\title{
Genetic identification of potential RNA-binding regions in a group II intron-encoded reverse transcriptase
}

\author{
SHAN-QING GU, ${ }^{1,2,3}$ XIAOXIA CUI, ${ }^{1,2,3,4}$ SIJIONG MOU, ${ }^{1,2,3}$ SABINE MOHR, ${ }^{1,2,3}$ JUN YAO, ${ }^{1,2,3}$ \\ and ALAN M. LAMBOWITZ1,2,3 \\ ${ }^{1}$ Institute for Cellular and Molecular Biology, University of Texas at Austin, Austin, Texas 78712-0159, USA \\ ${ }^{2}$ Department of Chemistry and Biochemistry, University of Texas at Austin, Austin, Texas 78712-0165, USA \\ ${ }^{3}$ Section of Molecular Genetics and Microbiology, School of Biological Sciences, University of Texas at Austin, Austin, Texas 78712-0182, USA
}

\begin{abstract}
Mobile group II introns encode a reverse transcriptase that binds the intron RNA to promote RNA splicing and intron mobility, the latter via reverse splicing of the excised intron into DNA sites, followed by reverse transcription. Previous work showed that the Lactococcus lactis LI.LtrB intron reverse transcriptase, denoted LtrA protein, binds with high affinity to DIVa, a stem-loop structure at the beginning of the LtrA open reading frame and makes additional contacts with intron core regions that stabilize the active RNA structure for forward and reverse splicing. LtrA's binding to DIVa down-regulates its translation and is critical for initiation of reverse transcription. Here, by using high-throughput unigenic evolution analysis with a genetic assay in which LtrA binding to DIVa down-regulates translation of GFP, we identified regions at LtrA's $\mathbf{N}$ terminus that are required for DIVa binding. Then, by similar analysis with a reciprocal genetic assay, we confirmed that residual splicing of a mutant intron lacking DIVa does not require these $\mathrm{N}$-terminal regions, but does require other reverse transcriptase (RT) and X/thumb domain regions that bind the intron core. We also show that N-terminal fragments of LtrA by themselves bind specifically to DIVa in vivo and in vitro. Our results suggest a model in which the $N$ terminus of nascent LtrA binds DIVa of the intron RNA that encoded it and nucleates further interactions with core regions that promote RNP assembly for RNA splicing and intron mobility. Features of this model may be relevant to evolutionarily related non-long-terminal-repeat (non-LTR)-retrotransposon RTs.
\end{abstract}

Keywords: retroelement; retrotransposon; ribozyme; RNA-protein interaction; translational control

\section{INTRODUCTION}

Mobile group II introns are retroelements found in bacteria and archaea as well as in eukarya in the organellar genomes of fungi, plants, protists, and annelid worms (for review, see Lambowitz and Zimmerly 2004). They consist of a catalytically active intron RNA ("ribozyme") and an intronencoded protein (IEP) with reverse transcriptase (RT) activity. The intron RNA contains six double-helical domains (DI-DVI), which fold into a conserved tertiary structure with an active site that uses specifically bound $\mathrm{Mg}^{2+}$ ions for catalysis (Pyle and Lambowitz 2006; Toor et al. 2008). This folded RNA catalyzes RNA splicing via two transesterification reactions that are the same as those of

${ }^{4}$ Present Address: Sigma Advanced Genetic Engineering Labs, 2033 Westport Center Drive, St. Louis, MO 63146, USA.

Reprint requests to: Alan $M$. Lambowitz, Institute for Cellular and Molecular Biology, University of Texas at Austin, Austin, TX 78712-0159, USA; e-mail: lambowitz@mail.utexas.edu; fax: (512) 232-3420.

Article published online ahead of print. Article and publication date are at http://www.rnajournal.org/cgi/doi/10.1261/rna.2007310. spliceosomal introns in higher organisms and yield ligated exons and an excised intron lariat (Peebles et al. 1986). For group II introns, the IEP, which is encoded in DIV, assists splicing by stabilizing the catalytically active RNA structure (Carignani et al. 1983; Moran et al. 1994; Matsuura et al. 2001). It then remains bound to the excised intron lariat RNA in a ribonucleoprotein particle (RNP) that promotes intron mobility (Zimmerly et al. 1995; Saldanha et al. 1999). Mobility occurs by a target DNA-primed reverse transcription mechanism in which the excised intron RNA reverse splices directly into a DNA strand and is reverse transcribed by the IEP, using either the cleaved opposite DNA strand or a nascent strand at a DNA replication fork to prime reverse transcription (Lambowitz and Zimmerly 2004).

Mobile group II introns are hypothesized to have played a pivotal role in genome evolution as ancestors of both spliceosomal introns and non-long-terminal-repeat (nonLTR) retrotransposons in higher organisms (Lambowitz and Zimmerly 2004; Pyle and Lambowitz 2006). An evolutionary relationship between group II and spliceosomal introns is suggested by the similarities in their splicing 
mechanisms, by structural and functional similarities between group II intron RNA domains and snRNAs, and by the ability of group II introns to be fragmented into functionally reassociating segments, suggesting an evolutionary origin for snRNAs (Sharp 1985, 1991; Cech 1986; Madhani and Guthrie 1992; Shukla and Padgett 2002; Toor et al. 2010).

An evolutionary relationship between group II introns and non-LTR retrotransposons is indicated by similarities in their RT sequences and retrotransposition mechanisms. The RTs of group II introns and non-LTR retrotransposons contain seven conserved sequence blocks (RT-1-RT-7) characteristic of all RTs, but differ from retroviral RTs in having an $\mathrm{N}$-terminal extension with conserved sequence block RT-0, as well as additional insertions in the RT and thumb domains, some with conserved structural features in group II intron and non-LTR-retrotransposon RTs (Xiong and Eickbush 1990; Malik et al. 1999; Blocker et al. 2005). Like group II intron RTs, non-LTR-retrotransposon RTs promote retrotransposition by using a target DNA-primed reverse transcription mechanism in which a cleaved DNA strand is used as a primer for reverse transcription of the element's RNA, and the cDNA initiation site is determined primarily by specific binding of the RNA template rather than by base pairing of a primer, as for retroviral RTs (Luan et al. 1993; Zimmerly et al. 1995). It has been speculated that the $\mathrm{N}$-terminal extension and/or other RT- and thumb-domain insertions in group II intron and non-LTR-retroelement RTs contribute to their distinctive properties, including higher processivity than that of retroviral RTs (Bibillo and Eickbush 2002a) and specific binding of the template RNA for initiation of reverse transcription (Chen and Lambowitz 1997; Bibillo and Eickbush 2002b; Blocker et al. 2005).

Studies with the Lactococcus lactis Ll.LtrB intron, which has been used as a model system, have revealed features of how group II intron RTs bind to the intron RNA to promote RNA splicing and intron mobility. The Ll.LtrB IEP, denoted LtrA protein, has four domains: RT, which contains the conserved RT sequence blocks and corresponds to fingers and palm regions of retroviral RTs; X, which corresponds to the RT thumb and is a site of mutations affecting RNA splicing activity; D, DNA binding; and En, DNA endonuclease (Cui et al. 2004; Blocker et al. 2005). The RT and X domains bind the intron RNA to stabilize its active structure for RNA splicing and reverse splicing, while the D and En domains are not required for RNA splicing but instead interact with DNA target sites during intron mobility (San Filippo and Lambowitz 2002; Cui et al. 2004; Blocker et al. 2005). LtrA appears to be largely monomeric in solution, but binds to the intron RNA with a stoichiometry of 2:1, suggesting that it functions as a dimer, similar to HIV-1 and some other RTs (Saldanha et al. 1999; Rambo and Doudna 2004).

Biochemical experiments showed that LtrA binds with high affinity to DIVa, a small stem-loop structure that contains the Shine-Dalgarno (SD) sequence and initiation codon at the beginning of the LtrA ORF, and makes additional contacts with conserved catalytic core regions, including parts of DI, DII, and DVI, which stabilize the active RNA structure (Wank et al. 1999; Matsuura et al. 2001; Dai et al. 2008). DIVa is thought to contribute to the intron specificity of binding, but if DIVa is deleted, LtrA can still promote RNA splicing at reduced efficiency by binding directly to the catalytic core (Wank et al. 1999; Matsuura et al. 2001). Notably, LtrA functions preferentially in cis to promote the splicing of the intron that encodes it (Cui et al. 2004), and the binding of LtrA to DIVa down-regulates its own translation (Singh et al. 2002). Although not always a site of translation initiation, a predicted DIVa stem-loop structure is present in most group II introns that encode IEPs, and the yeast aI2 IEP has also been shown to bind both DIVa and catalytic core regions, suggesting that this mode of interaction is conserved among group II IEPs (Singh et al. 2002; Huang et al. 2003). For Ll.LtrB, the binding of the IEP to DIVa was found to be critical for correct initiation of reverse transcription (Wank et al. 1999), and as expected from this finding, deletion of DIVa from Ll.LtrB or aI2 almost completely abolished intron mobility (D'Souza and Zhong 2002; Huang et al. 2003).

Previously, we used a genetic assay in which the splicing of the Ll.LtrB intron is linked to the expression of GFP in conjunction with a high-throughput genetic method termed "unigenic evolution" to identify regions of LtrA required for RNA splicing (Cui et al. 2004). Mapping of these regions onto a three-dimensional model of LtrA suggested an extended RNA-binding surface that spans the RT and X/thumb domains and includes the $\mathrm{N}$-terminal extension, regions in and around the template-primer binding track, and patches on the back of the molecule (Blocker et al. 2005). Deletion of the N-terminal extension strongly decreased LtrA's binding affinity for the intron RNA, but not LtrA-promoted RNA splicing, raising the possibility that the N-terminal extension interacts with DIVa. Conversely, a domain X mutant bound tightly to the intron RNA but was unable to promote RNA splicing, suggesting disruption of a critical interaction with catalytic core regions (Cui et al. 2004).

Here, we developed a new genetic assay to directly analyze the interaction of LtrA with DIVa by linking LtrA binding to DIVa to down-regulation of GFP expression. By using this genetic assay in conjunction with unigenic evolution analysis, we identified two regions at the $\mathrm{N}$ terminus of LtrA that are required for DIVa binding. Then, by unigenic evolution analysis using a reciprocal genetic assay, we confirmed that the residual splicing of a mutant intron lacking DIVa does not require these N-terminal regions, but does require other RT and X/thumb domain regions that bind directly to the catalytic core. We also show that $\mathrm{N}$-terminal fragments of LtrA that contain all or part of the RT domain are sufficient to bind DIVa in vivo and in vitro. Our results support a model in which the $\mathrm{N}$ terminus of 
a nascent LtrA protein binds to DIVa of the intron RNA that encodes it, thereby halting further translation and nucleating interactions with intron core regions, leading to RNP assembly for RNA splicing and intron mobility.

\section{RESULTS}

\section{An Escherichia coli genetic assay for the binding of the LtrA protein to DIVa}

To study the interaction between LtrA and DIVa, we developed an E. coli genetic assay in which the binding of LtrA to DIVa down-regulates the translation of a GFP reporter. This assay employs plasmid pBDG-LtrA (Fig. 1A), which uses the $\mathrm{P}_{\mathrm{BAD}}$ promoter to express the LtrA protein, followed by a cassette consisting of DIVa with the ATG and next 14 codons of the LtrA ORF linked in-frame to GFP. The $\mathrm{P}_{\mathrm{BAD}}$ promoter is regulated by the activator protein $\mathrm{AraC}$, which is encoded separately on the plasmid and binds L-arabinose to induce transcription from $\mathrm{P}_{\mathrm{BAD}}$ (Guzman et al. 1995). After induction with L-arabinose, the expressed LtrA protein binds DIVa to down-regulate GFP translation (GFP ${ }^{-}$ phenotype), while mutations that impair the binding of LtrA to DIVa allow GFP production ( $\mathrm{GFP}^{+}$phenotype). This configuration makes it possible for newly synthesized LtrA to bind DIVa rapidly after transcription to tightly down-regulate GFP translation, mimicking the situation in the native intron.

The DIVa segment cloned in pBDGLtrA is the same as in previously analyzed DIVa constructs (Fig. 1B; Singh et al. 2002; Watanabe and Lambowitz 2004; Materials and Methods). The secondary structure of this region deduced from chemical modification and SELEX experiments for LtrA binding consists of two stems regions, DIVa(ii) and DIVa(iii), separated by an asymmetric bulged loop containing the LtrA initiation codon (Fig. 1B; Watanabe and Lambowitz 2004). The SD sequence (AGGGAGG) is located a short distance upstream of the initiation codon spanning the DIVa(ii)/(iii) stems. Critical nucleotide residues for LtrA binding identified by SELEX analysis (boxed) are found in DIVa(ii)/(iii)

B
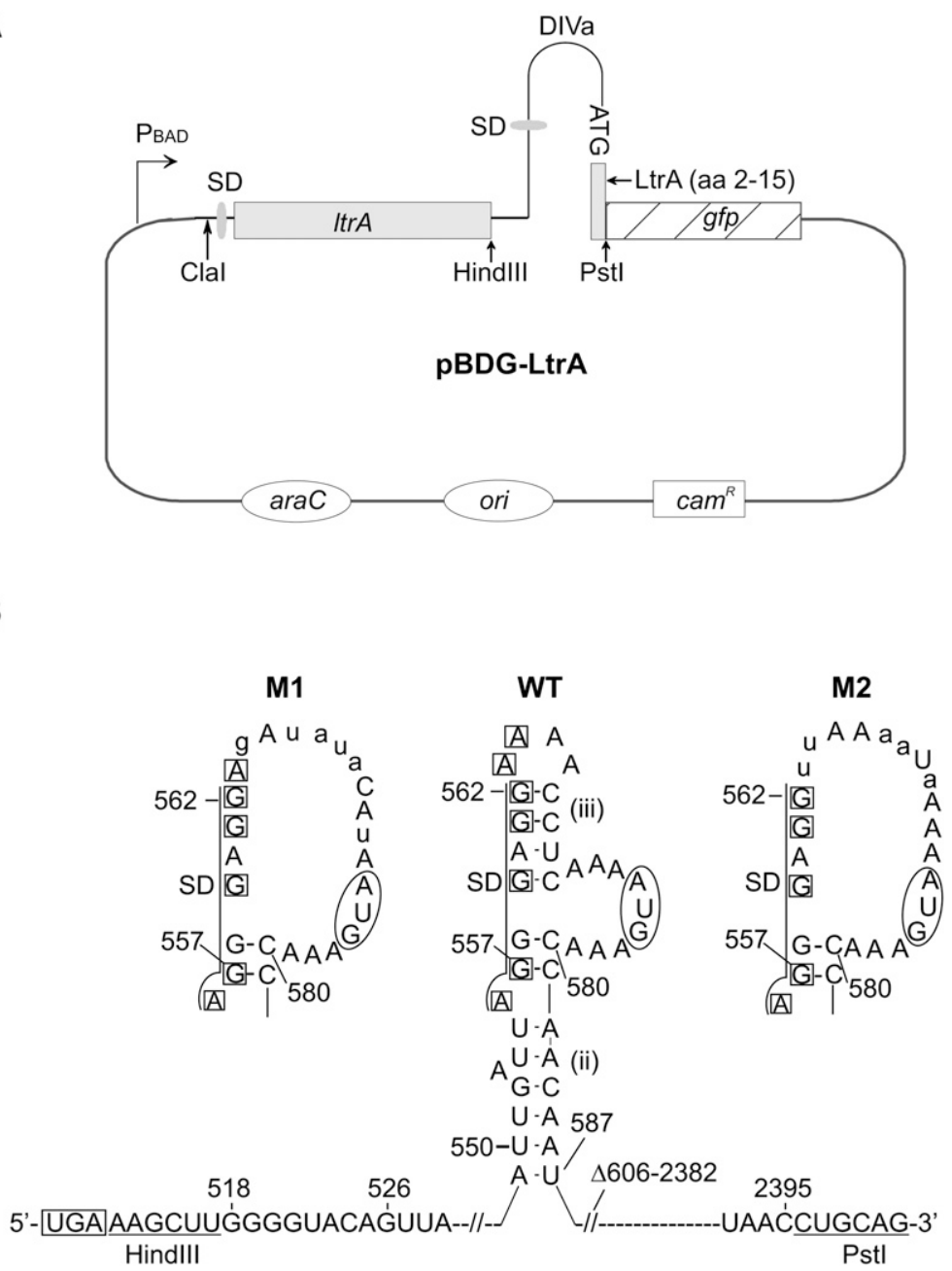

FIGURE 1. Genetic assay for the binding of the LtrA protein to the DIVa. (A) Plasmid pBDGLtrA used in the genetic assay. The plasmid employs an L-arabinose-inducible promoter $\left(\mathrm{P}_{\mathrm{BAD}}\right)$ to express the wild-type LtrA protein, followed by a cassette consisting of DIVa with the Shine-Dalgarno (SD) sequence, ATG initiation codon, and next 14 codons of the LtrA ORF fused in-frame to codon 2 of GFP. In the genetic assay, the LtrA protein binds DIVa to downregulate translation of GFP, leading to a $\mathrm{GFP}^{-}$phenotype, while mutations that impair binding of LtrA to DIVa lead to a $\mathrm{GFP}^{+}$phenotype. The plasmid encodes the activator protein AraC, which binds L-arabinose to induce expression from the $\mathrm{P}_{\mathrm{BAD}}$ promoter. $\left(\mathrm{cam}^{R}\right)$ Chloramphenicol-resistance marker; (ori) plasmid replication origin. The region containing DIVa is expanded to emphasize structural features. (B) DIVa region of pBDG-LtrA and changes in DIVa mutants M1 and M2. The secondary structure of the LtrA-binding region of wild-type (WT) DIVa was deduced from in vitro selection experiments (Singh et al. 2002; Watanabe and Lambowitz 2004). Nucleotide residues found to be critical for LtrA binding in those experiments are boxed. The SD sequence is delineated, and the AUG initiation codon is circled. The insets at left and right show changes in DIVa mutants M1 and M2, with mutant nucleotide residues shown in lowercase letters. The UGA termination codon of the LtrA ORF located upstream of DIVa (see $A$ ) is boxed. The discontinuity of intron position numbers downstream from DIVa reflects deletion of the intron ORF. The locations of some restriction sites used for cloning are shown (see Materials and Methods). overlapping the SD sequence and in the terminal DIVa tetraloop (Singh et al. 2002; Watanabe and Lambowitz 2004). The binding of LtrA occludes the SD sequence, either directly or by stabilizing the $\mathrm{DIVa}(\mathrm{ii}) /(\mathrm{iii})$ stems, thereby down-regulating translation (Singh et al. 2002). 
For the genetic assays, pBDG-LtrA or derivatives containing specific modifications are transformed into E. coli $\mathrm{DH} 5 \alpha$, an $\mathrm{ara}^{-}$strain that is dependent upon addition of exogenous L-arabinose to induce LtrA and GFP expression. The synthesis of GFP is monitored either by a plating assay in which colonies are lifted to nitrocellulose filter, induced with L-arabinose, and scanned by using a PhosphorImager, or for cells grown in liquid culture by using a fluorescenceactivated cell sorter (FACS) assay. In both assays, cells are grown in $\mathrm{LB}$ medium and induced with $1 \% \mathrm{~L}$-arabinose overnight at $30^{\circ} \mathrm{C}$.

Figure 2 shows results for an initial series of test constructs assayed in parallel. In both the plating and FACS assays, pBDG-LtrA, which encodes the wild-type LtrA protein, did not give detectable fluorescence above background (Fig. 2A), while pBDG, an otherwise identical construct that does not encode LtrA, gave strong GFP fluorescence (Fig. 2B). Thus, LtrA down-regulates GFP expression in these assays. Two DIVa mutations in which the critical 11-nucleotide (nt) region between the SD sequence and AUG codon was replaced by different sequences, gave increased GFP fluorescence, as expected for impaired binding of LtrA to DIVa (Fig. 2C,D, M1, M2). These relatively large mutations, which change the tetraloop sequence as well as disrupt the DIVa(iii) stem, were necessary because smaller mutations in the tetraloop, such as replacement of the first two A-residues with C-residues, were by themselves insufficient to alleviate translational repression by LtrA (data not shown). Although the latter mutations strongly decreased LtrA binding in assays done

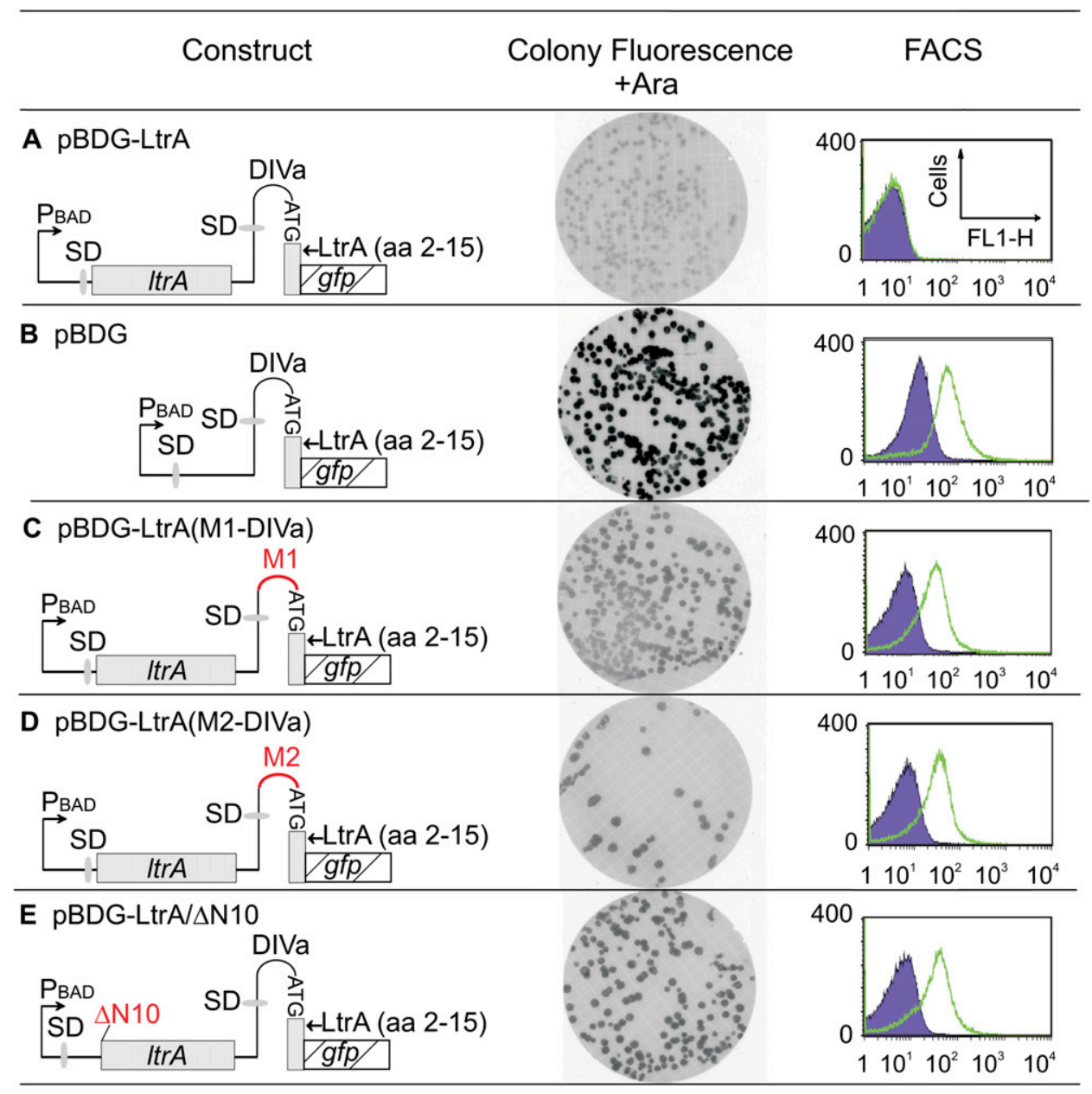

FIGURE 2. Wild-type and mutant constructs assayed for DIVa binding by plating and FACS assays for GFP expression. E. coli DH5 $\alpha$ containing pBDG-LtrA or mutants diagrammed schematically to the left were induced with L-arabinose (+Ara), and GFP fluorescence was monitored by plating and FACS assays, as described in the Materials and Methods. The plating assays for the different constructs were done in parallel. The FACS profiles show the numbers of cells in a window of fluorescence intensity before (purple) and after (green) L-arabinose induction. The constructs tested were: $(A)$ pBDG-LtrA; $(B)$ pBDG, identical to pBDG-LtrA but lacking the LtrA ORF; $(C, D)$ pBDG-LtrA(M1-DIVa) and pBDG$\mathrm{LtrA}(\mathrm{M} 2-\mathrm{DIVa})$, mutants in which the sequence $5^{\prime}$-AAAACCTCAA between the SD sequence and initiation codon in DIVa was replaced by 5 -AgAtataCAt and 5'-ttAAaaTaAA, respectively (see Fig. 1; lowercase letters indicate mutant nucleotide residues). (E) pBDG-LtrA/ $\Delta$ N10, which expresses a mutant LtrA protein with an $\mathrm{N}$-terminal truncation of 10 amino acid residues. Mutated regions are shown in red. 
in vitro at high monovalent salt concentration to maintain LtrA solubility and specificity (Singh et al. 2002), they have less effect in the in vivo assay, likely reflecting that electrostatic interactions with the phosphodiester backbone make a greater contribution to LtrA binding at lower salt concentrations in vivo. Finally, a construct expressing a mutant LtrA protein with a 10 -amino acid residue N-terminal truncation (LtrA/ $\Delta \mathrm{N} 10)$ gave strong GFP fluorescence, indicating impaired DIVa binding (Fig. 2E, pBDG-LtrA/ $\Delta \mathrm{N} 10)$. Immunoblots confirmed that $\operatorname{LtrA} / \Delta \mathrm{N} 10$ is expressed from this construct at levels comparable to the wild-type LtrA protein (Supplemental Fig. S1).

\section{Unigenic evolution analysis for DIVa binding}

To systematically identify regions of LtrA important for DIVa binding, we used the GFP-expression assay in combination with a high-throughput method termed unigenic evolution analysis (Deminoff et al. 1995; Cui et al. 2004; Mohr et al. 2008). This method involves isolating a collection of functional protein variants from a mutant library, and then statistically analyzing ratios of missense to silent mutations to assess the degree of constraint on different protein regions. The latter is expressed by a parameter termed the mutability value $(\mathrm{M})$, with negative and positive $M$ values indicating hypomutability and hypermutability, respectively (see Materials and Methods). To analyze LtrA binding to DIVa, we constructed a pBDG-LtrA library in which random mutations were introduced into the LtrA ORF by mutagenic PCR. Sequencing of 36 randomly selected plasmids from the library showed the mutation frequency was $0.54 \%$, and the average number of amino acid substitutions per protein was 7.3.

To identify functional protein variants, the library was transformed into E. coli DH5 $\alpha$ and screened by colony fluorescence assays to identify those with low GFP fluorescence similar to that of wild-type LtrA. Such colonies, which putatively express LtrA variants that bind DIVa, were grown up in liquid culture, and plasmids were isolated and retransformed into DH5 $\alpha$ for FACS assays to confirm the $\mathrm{GFP}^{-}$ phenotype. Those plasmids that gave $<15 \%$ of the fluorescence increase of the control plasmid pBDG, which does not encode LtrA, were sequenced to identify the mutations.

We identified 107 LtrA variants that met the above criteria for a $\mathrm{GFP}^{-}$phenotype and harbor plasmids encoding full-length LtrA without premature stop codons. These variants contained a total of 582 mutations, of which 442 were missense and 140 were silent. The mutations are summarized in Supplemental Figure S2, and a mutability plot based on the data is shown in Figure 3.

The mutability plot identified two significantly hypomutable regions at the $\mathrm{N}$ terminus of LtrA that are required for binding DIVa. The first hypomutable region (Fig. 3, region A, amino acid residues $13-41, P=0.0036)$ corresponds to the $\mathrm{N}$-terminal extension, and the second hypo-

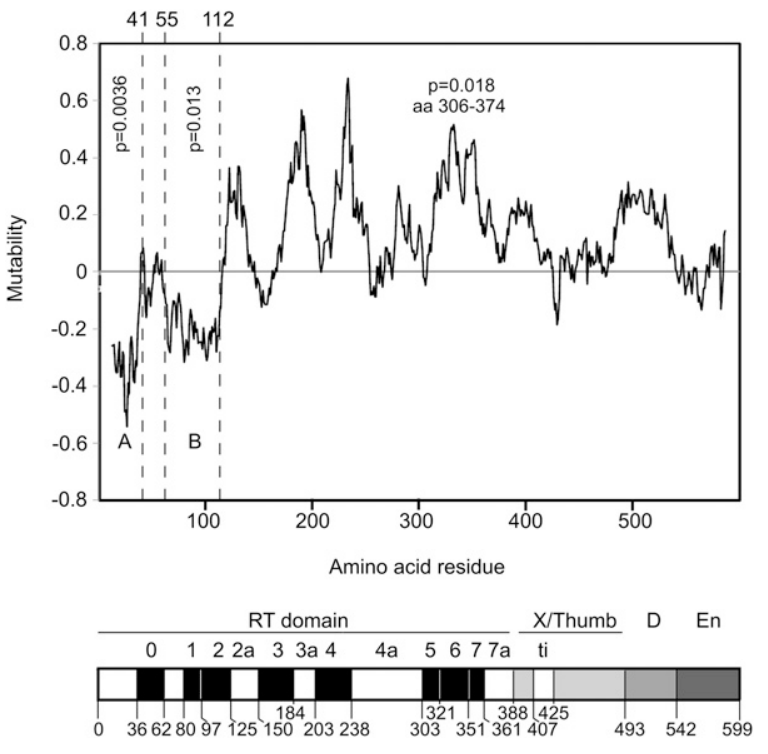

FIGURE 3. Unigenic evolution analysis of LtrA variants that bind DIVa. E. coli $\mathrm{DH} 5 \alpha$ containing a pBDG-LtrA library with random PCRinduced mutations in the LtrA ORF was induced with L-arabinose and screened by using a colony-based fluorescence assay to identify functional LtrA variants that bind DIVa to down-regulate GFP expression $\left(\mathrm{GFP}^{-}\right.$phenotype). Plasmids isolated from putative $\mathrm{GFP}^{-}$colonies were retransformed into DH5 $\alpha$ for FACS assay to confirm the GFP ${ }^{-}$ phenotype (defined as $<15 \%$ of the L-arabinose-induced GFP fluorescence increase in a parallel FACS assays with the control plasmid pBDG), and then sequenced to identify mutations. The plot shows mutability $(\mathrm{M})$ values at the center of a 25 -amino acid sliding window based on the sequences of 107 functional LtrA variants that showed a $\mathrm{GFP}^{-}$phenotype and contained 442 missense and 140 silent mutations (summarized in Supplemental Fig. S2). Negative M-values indicate hypomutable regions, with a minimum value of -1 indicating no missense mutations in the 25 -amino acid residue window, and positive $\mathrm{M}$-values indicate hypermutability normalized to a maximum value of +1 . $P$-values are shown for hypomutable regions $A$ and $B$, and the statistically significant hypermutable region (amino acid residues 306-374). Although two other peaks have higher M-values suggesting hypermutability, they are not statistically significant based on the numbers of missense and silent mutations observed in these regions compared with those expected ( $P>0.17$; see Materials and Methods). A schematic of LtrA showing different regions aligned with the mutability plot is below. RT-1 to RT-7 are conserved sequence blocks characteristic of all RTs, and RT-0, RT-2a, RT-3a, RT-4a, RT-7a, and ti are RT or thumb domain insertions found in group II intron RTs (Blocker et al. 2005).

mutable region (Fig. 3, region B, amino acid residues 55$112, P=0.013$ ) extends from the end of RT-0 to RT-2 (Fig. 3 , bottom). Notably, RT-0 itself appears less constrained in this assay than the regions flanking it. Other regions of the protein were not significantly hypomutable and are thus not required for DIVa binding in this assay. Some of these other regions have positive mutability values, suggesting that they may be hypermutable, but only one of these positive peaks (Fig. 3, positions 306-374, RT-5-RT-7a) was statistically significant based on this data set (Fig. 3, $P=$ 0.018). Hypermutability, if real, could reflect selection for mutations that stabilize the protein or promote folding of 
regions $\mathrm{A}$ or $\mathrm{B}$, which are identified by the unigenic evolution analysis as being required for DIVa binding.

\section{In vivo analysis of DIVa binding by N-terminal segments of LtrA}

Surprisingly, the unigenic evolution analysis identified an additional $25 \mathrm{GFP}^{-}$variants containing premature stop codons, including at positions 80, 85, 97, 149, and 154 (Supplemental Fig. S2, asterisks). These variants were analyzed by FACS and confirmed to be $\mathrm{GFP}^{-}$. The premature stop codons in these variants raised the possibility that N-terminal fragments of LtrA alone might be functional for DIVa binding. However, because of the geometry of the construct, the $\mathrm{GFP}^{-}$phenotype could also reflect polarity due to premature termination of translation within the LtrA ORF, resulting in degradation of the downstream mRNA encoding GFP.

To test systematically whether N-terminal fragments of LtrA could bind DIVa in vivo, we constructed a series of derivatives of pBDG-LtrA expressing different C-terminally truncated LtrA proteins with two consecutive stop codons (TGA, TAA) at the site of the truncation, followed immediately by the DIVa cassette as in the wild-type pBDG-LtrA plasmid (see Fig. 1B). Thus, each N-terminal LtrA segment is tested with the identical downstream sequence. When expressed from these constructs, LtrA variants terminated at codon 80 or 150 gave high GFP fluorescence, indicating failure to bind DIVa, while an LtrA variant terminated at codon 202 gave somewhat decreased GFP fluorescence, and LtrA variants terminated at codons $237,303,320$, or 365 gave no GFP fluorescence above background, similar to the wild-type protein (Fig. 4A,B). SDS-PAGE and immunoblotting using an anti-LtrA antibody that fortuitously detects both LtrA and the short LtrA segment fused to the $\mathrm{N}$ terminus of GFP showed directly that the expression of the GFP protein is not affected by LtrA/ $\Delta 150-599$, which ends after RT-2a, is decreased by LtrA/ $\Delta 202-599$, which ends after RT-3a, and reduced to background levels by LtrA/ $\Delta 237-599$ and other truncations, which end after RT-4 (Fig. 4C). Together, these findings show that $\mathrm{N}$-terminal fragments of LtrA can bind DIVa in vivo. The finding that the $\mathrm{N}$-terminal fragments must extend through RT-4 for full DIVa-binding activity rather than RT-2, the end of region $\mathrm{B}$ identified in the unigenic evolution analysis, may reflect that additional downstream sequences are required for stability of the protein and/or correct folding of the N-terminal DIVabinding regions (regions $\mathrm{A}$ and/or $\mathrm{B}$ ).

\section{Unigenic evolution analysis to identify regions of LtrA required for splicing an LI.LtrB- $\Delta$ DIVa intron}

An important prediction of our model is that regions of LtrA that function only in DIVa binding should not be required for the splicing of a mutant Ll.LtrB- $\Delta$ ORF intron lacking DIVa, which occurs inefficiently by the direct binding of LtrA to core regions of the intron RNA (Wank et al. 1999; Cui et al. 2004). To test this prediction, we used a previously developed E. coli genetic assay, in which the splicing of wild-type or mutant Ll.LtrB- $\triangle$ ORF introns is linked to the expression of GFP (Cui et al. 2004). For this assay, we constructed plasmid pELG2- $\triangle D I V a$, which uses a T7lac promoter to express a cassette consisting of the Ll.LtrB- $\Delta$ DIVa intron (previously denoted Ll.LtrB- $\Delta$ ORF/ $\Delta$ DIVa) (Cui et al. 2004) and flanking exons, with exon 2 linked in frame to codon 2 of GFP (Fig. 5A). The LtrA protein is expressed from the same plasmid from a position downstream from the GFP ORF. Because LtrA-promoted splicing of the Ll.LtrB- $\Delta$ DIVa intron is inefficient and results in only weak GFP fluorescence, we identified splicing-competent LtrA variants by a more sensitive colony immunoblot assay in which GFP expression in plated colonies is monitored by using an anti-GFP antibody (Fig. 5B).

To identify regions of LtrA required for splicing Ll.LtrB$\triangle \mathrm{DIVa}$, we carried out unigenic evolution analysis using a pELG2- $\triangle$ DIVa library with random LtrA mutations induced by mutagenic PCR. LtrA variants that could splice the intron were identified by the colony immunoblot assay. The plasmids were then isolated and retransformed into E. coli HMS174(DE3) to confirm splicing by RT-PCR (data not shown) and sequenced to identify the mutations. We sequenced 170 splicing-competent LtrA variants that satisfied these criteria and found them to contain a total of 945 mutations, of which 575 were missense and 370 were silent. The mutations are summarized in Supplemental Figure S3, and a mutability plot based on the data is shown in Figure 6. A mutability plot for LtrA-promoted splicing of the wildtype Ll.LtrB- $\triangle$ ORF intron based on previous data (Cui et al. 2004) is superimposed for comparison (Fig. 6, dotted line) and shown separately in Supplemental Figure S4.

First, the plots show that the regions $\mathrm{A}$ and $\mathrm{B}$ at the $\mathrm{N}$ terminus of LtrA, which are required for binding DIVa (Fig. 3) and for efficient splicing of the wild-type Ll.LtrB$\Delta$ ORF intron (Fig. 6, dotted line; Supplemental Fig. S4), are not significantly hypomutable in variants that promote splicing of the Ll.LtrB- $\Delta \mathrm{DIVa}(P$-values for the small negative peaks are 0.86 and 0.22 , respectively), indicating that they are not required for residual splicing of the mutant intron lacking DIVa. Thus, the plot confirms the prediction that these $\mathrm{N}$-terminal regions are required primarily for DIVa binding.

Second, the plot identifies three regions in the RT and $\mathrm{X} /$ thumb domains, $\mathrm{C}, \mathrm{E}$, and $\mathrm{F}$, which are significantly hypomutable in variants that function in Ll.LtrB- $\Delta$ DIVa intron splicing (Fig. 6, region $\mathrm{C}$ [positions 112-173, $P=$ 0.0025], region $\mathrm{E}$ [positions 326-415, $P=0.0004$ ], region $\mathrm{F}$ [positions 418-500, $P=0.0097$ ]). These regions overlap those needed for splicing the wild-type Ll.LtrB- $\Delta$ ORF 
A

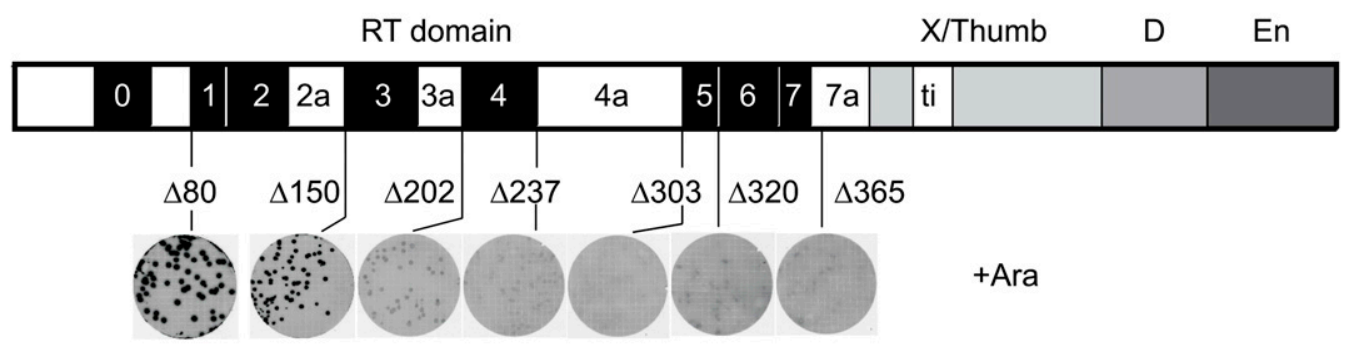

B

pBDG-LtrA/ $\Delta 150-599$

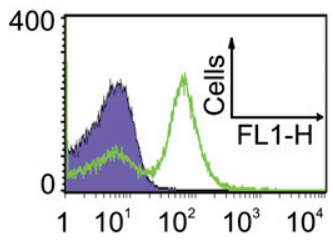

pBDG-LtrA/ $\Delta 237-599$

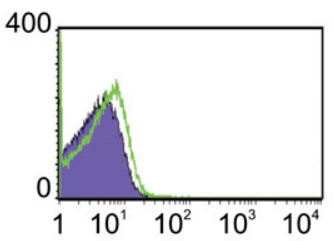

pBDG-LtrA/ $\Delta 320-599$

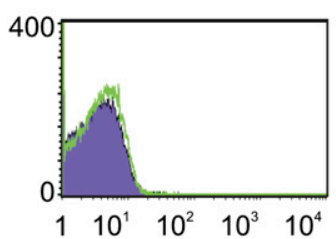

pBDG-LtrA/ $\Delta 202-599$

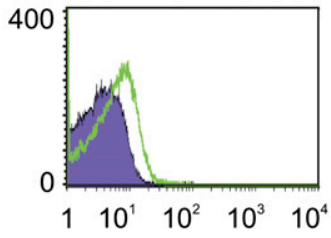

pBDG-LtrA/ $\Delta 303-599$

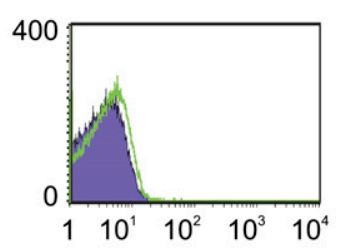

C

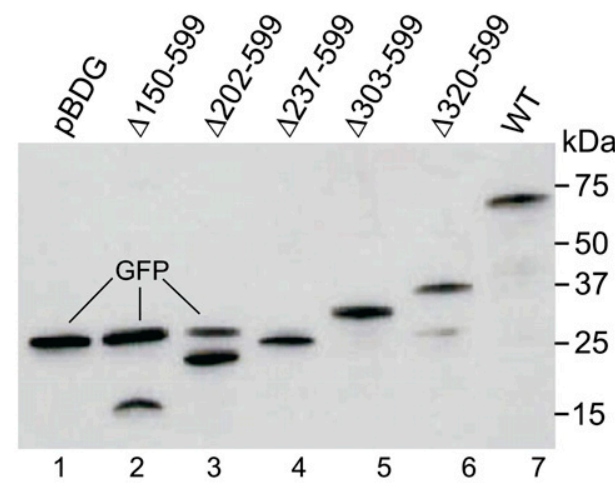

pBDG-LtrA/ $\Delta 365-599$

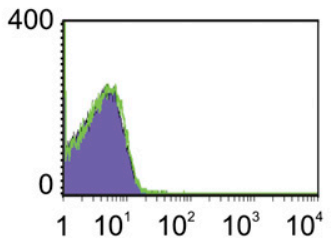

FIGURE 4. In vivo assays of DIVa binding by C-terminally truncated LtrA proteins. E. coli DH5 $\alpha$ containing pBDG-LtrA expressing wild-type LtrA or the indicated C-terminal truncation mutants was induced with $1 \%$ L-arabinose (+Ara) and analyzed for GFP expression by plating and FACS assays and for protein expression by SDS-PAGE and immunoblotting with an anti-LtrA antibody. (A) Schematic of LtrA protein showing the location of C-terminal truncations, and plating assays for GFP fluorescence shown below. The plating assays for different mutants were done in parallel. (B) FACS assays. The FACS profiles show the number of cells in a fluorescence window before (purple) and after (green) induction with L-arabinose. Data are representative of at least two independent repeats for each construct. (C) Immunoblot analysis. SDS-PAGE and immunoblotting with anti-LtrA antibody were done as described in the Materials and Methods. Positions of Precision plus protein standards (Bio-Rad) are shown to the right of the blot. Equal loading was confirmed by Coomassie blue staining of a parallel gel (data not shown).

intron and are presumably required for interaction with core regions of the intron RNA.

Finally, comparison of the plots identifies one region (Fig. 6, region D) that was not identified as being required for binding DIVa in the initial unigenic evolution analysis (Fig. 3) and does not contribute to splicing the Ll.LtrB$\Delta$ DIVa intron (Fig. 6), but is required for efficient splicing of the wild-type Ll.LtrB- $\Delta$ ORF intron (Fig. 6, dotted line; Supplemental Fig. S4). This region, extending from RT-3 to RT-4, is the same as that identified above as being required in addition to regions $\mathrm{A}$ and $\mathrm{B}$ for DIVa by $\mathrm{N}$-terminal LtrA fragments (Fig. 4). Thus, its requirement for efficient splicing of the wild-type Ll.LtrB- $\Delta \mathrm{ORF}$ intron may reflect that it enhances DIVa binding by stabilizing the fold of regions $\mathrm{A}$ and $\mathrm{B}$. It could also contribute to efficient splicing of the wild-type Ll.LtrB- $\Delta$ ORF intron by contacting other regions of the intron RNA in a DIVa-dependent manner.

\section{Biochemical assays of DIVa binding}

Finally, to confirm the results of the genetic assays, we tested directly whether $\mathrm{N}$ - and C-terminal truncation mutants of LtrA could bind specifically to DIVa in vitro. Two N-terminally truncated LtrA proteins (LtrA/ $\Delta \mathrm{N} 10$ and $\mathrm{LtrA} / \Delta \mathrm{N} 20)$ and two C-terminally truncated proteins 
A
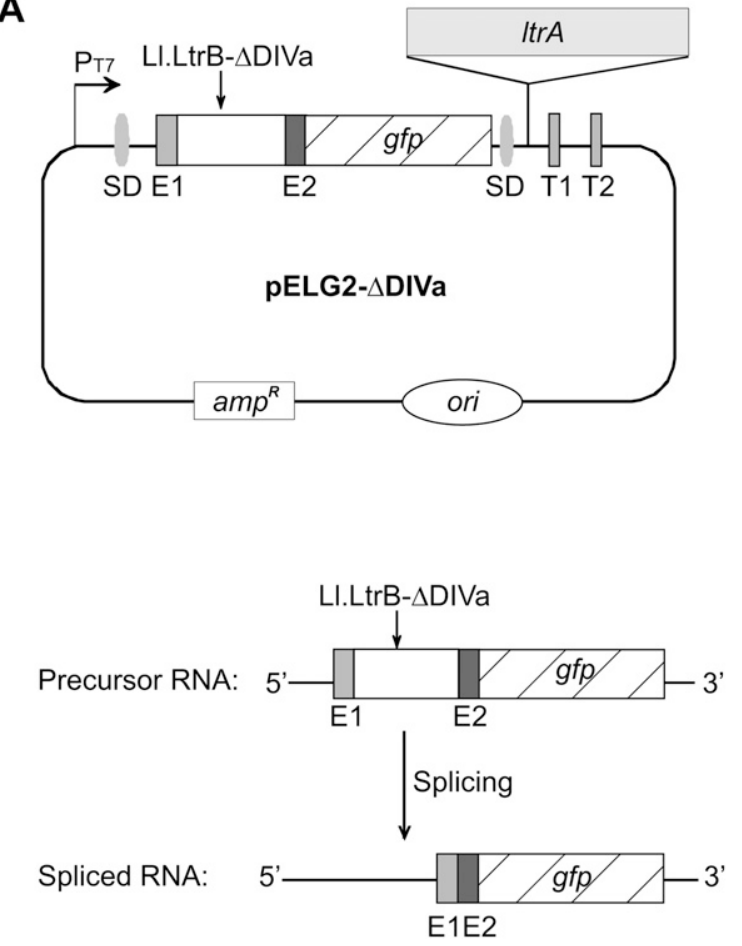

B

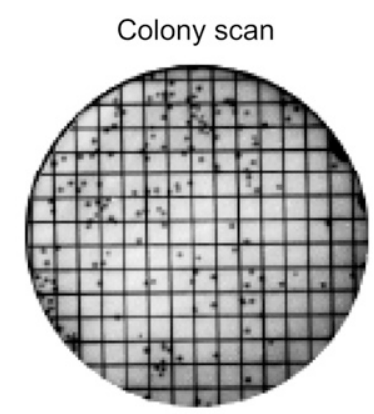

Colony immunoblot

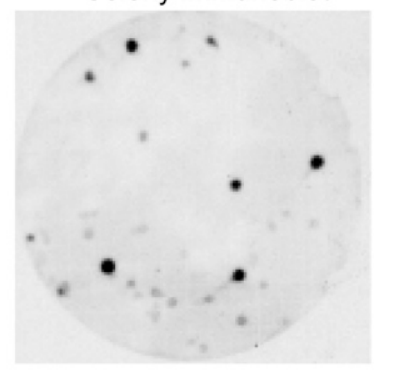

FIGURE 5. Genetic assay of LtrA-promoted splicing of the Ll.LtrB- $\Delta$ DIVa intron and colony-based immunoblot assay for GFP expression. ( $A$ ) Map of plasmid pELG2- $\triangle$ DIVa used in the genetic assay. The plasmid contains an ltrB/GFP fusion cloned downstream from a T7lac promoter (arrow) and phage T7 $\phi 10$ gene Shine-Dalgarno (SD) sequence. The ltrB/GFP fusion is comprised of the first eight codons of the $\phi 10$ gene fused to a segment of the L. lactis ltrB gene consisting of the Ll.LtrB- $\Delta$ DIVa intron (open rectangle) and flanking exons (E1 and E2; filled boxes), with E2 linked in-frame to the GFP coding sequence (hatched). The LtrA ORF is cloned downstream from the GFP ORF. T1 and T2 are E. coli rrnB transcription terminators. The schematic below shows that splicing of the intron leads to the expression of GFP with a short N-terminal extension (42 amino acid residues) corresponding to the $\phi 10$ sequence and ligated exons fused to GFP. In the absence of splicing, translation is terminated by stop codons within the intron. (B) Colony-based immunoblot assay for GFP expression. E. coli HMS174(DE3) containing pELG2- $\Delta$ DIVa with and without $\operatorname{ltr}$ (splicing competent and splicing defective, respectively) were mixed at a ratio of 1:10, then analyzed for GFP expression by colony immunoblot assay, as described in the Materials and Methods. The top shows an optical scan of the filter before treatment, and the bottom shows a chemiluminescence exposure of the filter after staining with anti-GFP antibody. Splicing-competent colonies producing GFP appear black on a light background.

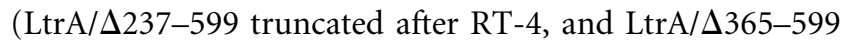
truncated after RT-7) were expressed and purified using the same intein-based expression and purification system used to express and purify the full-length LtrA protein (Saldanha et al. 1999). The LtrA/ $\Delta 237-599$ protein contains the minimal DIVa-binding region identified by the in vivo assays (Fig. 4). The proteins were more than 95\% pure (Supplemental Fig. S5). Biochemical assays showed that the C-terminally truncated proteins LtrA/ $\Delta 237-599$ and LtrA/ $\Delta 365-599$ lacked both RT and RNA splicing activity, as expected, because they are missing part of the RT domain and/or the $\mathrm{X} /$ thumb domain, while the $\mathrm{N}$-terminally truncated proteins LtrA/ $\Delta \mathrm{N} 10$ and LtrA/ $\Delta \mathrm{N} 20$ retained high RT and RNA splicing activity, in agreement with previous biochemical assays of these proteins (Supplemental Figs. S6, S7; Cui et al. 2004).

First, we carried out equilibrium-binding assays, in which the purified proteins were incubated with ${ }^{32} \mathrm{P}$-labeled DIVa RNA in reaction medium containing $450 \mathrm{mM} \mathrm{NaCl}$ and $5 \mathrm{mM} \mathrm{Mg}^{2+}$ (NMT) (see Materials and Methods) for
$60 \mathrm{~min}$ at $30^{\circ} \mathrm{C}$ and then filtered through a nitrocellulose membrane to bind RNA-protein complexes. The high salt concentration was found previously to be necessary for the specific binding of wild-type LtrA to the Ll.LtrB- $\Delta$ ORF intron (Saldanha et al. 1999). As shown in Figure 7A, wildtype LtrA binds DIVa with an apparent $K_{\mathrm{d}}=0.62 \mathrm{nM}$; the C-terminally truncated proteins LtrA/ $\Delta 237-599$ and LtrA/ $\Delta 365-599$ bind almost as strongly (apparent $K_{\mathrm{d}}=1.1$ and $1.0 \mathrm{nM}$, respectively); and the $\mathrm{N}$-terminally truncated proteins $\Delta \mathrm{N} 10$ and $\Delta \mathrm{N} 20$, which have small deletions in the $\mathrm{N}$-terminal extension, have substantially higher apparent $K_{\mathrm{d}}$ 's ( 4.5 and $20 \mathrm{nM}$, respectively). These equilibriumbinding assays do not distinguish between specific and nonspecific binding and may exaggerate nonspecific binding due to the large excess of LtrA protein.

To assess the specificity of binding of the truncated proteins to DIVa, we used a competition-binding assay (Fig. 7B). In this assay, wild-type and mutant LtrA proteins $(20 \mathrm{nM})$ were incubated with an excess $(100 \mathrm{nM}$ each) of two different-sized ${ }^{32} \mathrm{P}$-labeled RNAs, one a 135-nt RNA 


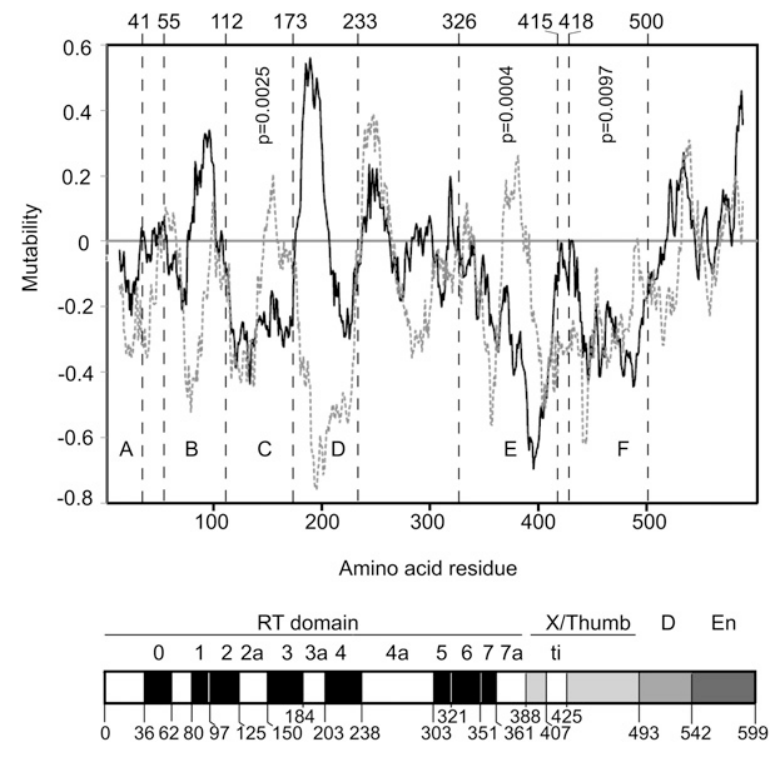

FIGURE 6. Unigenic evolution analysis of LtrA variants that splice the Ll.LtrB- $\Delta$ DIVa intron. E. coli HMS174(DE3) containing a pELG2$\triangle$ DIVa library with random PCR-generated mutations in the LtrA ORF was screened by colony immunoblot assay to identifying functional LtrA variants that promote splicing of the Ll.LtrB- $\Delta$ DIVa intron, leading to expression of GFP. Positive colonies were grown up in LB medium, and plasmids were isolated, retransformed into E. coli HMS174(DE3) to confirm splicing activity by RT-PCR for ligatedexons sequences, and sequenced to identify mutations. The plot shows mutability $(\mathrm{M})$ values at different positions in the LtrA protein calculated across a 25-amino acid residue sliding window, as described in the Materials and Methods. The analysis is based on sequences of 170 functional LtrA variants that could promote splicing of the Ll.LtrB- $\Delta$ DIVa intron and contained 945 mutations, of which 575 were missense and 370 were silent (summarized in Supplemental Fig. S3). Negative $\mathrm{M}$-values indicate hypomutability, with a minimum value of -1 indicating no missense mutations, and positive $\mathrm{M}$-values indicating hypermutability. $P$-values for statistically significant hypomutable regions are shown. A plot for LtrA variants that efficiently splice the wild-type Ll.LtrB- $\Delta$ ORF intron based on the unigenic evolution data in Cui et al. (2004) is shown as a dotted line for comparison (see Supplemental Fig. S4 for details). A schematic of LtrA showing different regions aligned with the mutability plot is shown below.

containing wild-type DIVa (control, C) and the other a 119-nt RNA containing wild-type or mutant DIVa (experimental, E). The DIVa mutants used in these experiments have nucleotide substitutions in the DIVa tetraloop shown previously to impair LtrA binding in vitro (wildtype AAAA changed to AgAA or gAAA, where the lowercase letter indicates the mutant nucleotide residue) (Singh et al. 2002). After incubation, the mixture was filtered through nitrocellulose, and the RNAs bound by the protein were eluted to assess the specificity of DIVa binding. The wild-type and both LtrA C-terminal truncations behaved similarly, showing equal binding of the two different-sized wild-type DIVa RNAs and preferential binding of the wildtype compared with the mutant DIVa RNAs with altered tetraloop sequences. These findings show that $\mathrm{N}$-terminal fragments of LtrA truncated after RT-4 (LtrA/ $237-599)$ or
RT-7 (LtrA/ $\Delta 365-599)$ bind DIVa in part by recognizing the tetraloop sequence similarly to wild-type LtrA.

In contrast, the N-terminally truncated LtrA proteins, $\operatorname{Ltr} \mathrm{A} / \Delta \mathrm{N} 10$, and $\operatorname{Ltr} \mathrm{A} / \Delta \mathrm{N} 20$, which lack only the first 10 or 20 amino acid residues of LtrA's $\mathrm{N}$-terminal extension, showed no specific binding to DIVa in this assay (Fig. 7B). As these proteins retain relatively high RT and RNA splicing activity (Cui et al. 2004; Supplemental Figs. S6, S7), the results suggest that all or part of the $\mathrm{N}$-terminal extension of LtrA upstream of RT-0 constitutes a separate functional domain that is required primarily for DIVa binding.

\section{DISCUSSION}

Here, we analyzed a group II intron RT, the LtrA protein encoded by the Ll.LtrB intron, by using high-throughput genetic methods to identify regions that are required for binding different parts of the intron RNA. First, unigenic evolution analysis with an $E$. coli genetic assay in which LtrA binding to DIVa down-regulates GFP translation identified two N-terminal regions of LtrA that are required for DIVa-binding in vivo (Fig. 3, regions A,B). Region A corresponds to the $\mathrm{N}$-terminal extension up to RT-0, while region $\mathrm{B}$ extends from RT-0 to RT-2. The further findings that $\mathrm{N}$-terminal fragments of LtrA containing regions $\mathrm{A}$ and B bind specifically to DIVa in vitro and in vivo (Figs. 4, 7) indicate that one or both of these regions functions directly in DIVa binding. Additional unigenic evolution analyses showed that regions $\mathrm{A}$ and $\mathrm{B}$ are required for efficient splicing of the wild-type Ll.LtrB- $\Delta$ ORF intron, as expected from their role in high-affinity binding to DIVa, but not for residual splicing of the Ll.LtrB- $\triangle$ DIVa intron (Fig. 6), which occurs by direct binding of LtrA to core regions of the intron RNA. The importance of LtrA's N-terminal extension (region A) for DIVa binding is underscored by the finding that $\mathrm{N}$-terminal truncations of only 10 or 20 amino acid residues abolish specific binding of DIVa in vivo and in vitro, while leaving relatively high RT and RNA splicing activity. The latter findings strongly suggest that the $\mathrm{N}$-terminal extension contacts DIVa directly.

Beside regions $\mathrm{A}$ and $\mathrm{B}$, we identified a third LtrA region that may enhance its binding to DIVa (Fig. 6, region D). This region, which extends from RT-3 to RT-4, was not hypomutable in the initial unigenic evolution analysis for DIVa binding by full-length LtrA, but was required along with regions $\mathrm{A}$ and $\mathrm{B}$ for DIVa binding by $\mathrm{N}$-terminal fragments of LtrA in vivo (Fig. 4). An N-terminal segment of LtrA truncated after RT-4 also bound specifically to DIVa in vitro (Fig. 7). Together, these findings suggest that region $\mathrm{D}$ must be present to stabilize the protein and/or for correct folding of regions A and/or B, one or both of which contact DIVa directly. Region $\mathrm{D}$ was also found by unigenic evolution analysis to be hypomutable in variants that efficiently splice the wild-type Ll.LtrB- $\Delta$ ORF intron, consistent with a role in enhancing DIVa binding by stabilizing 
A

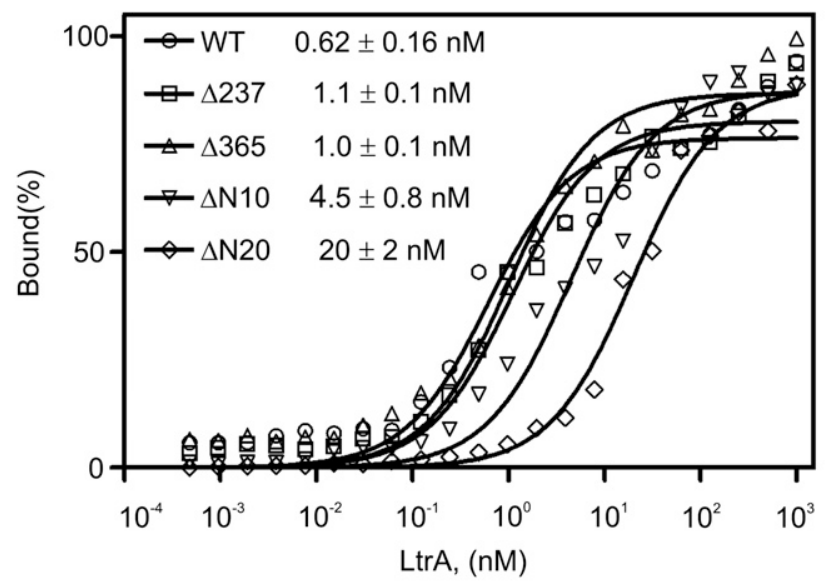

B Input

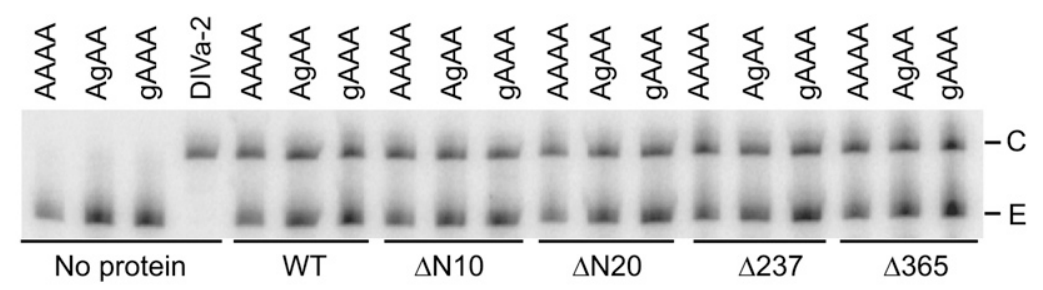

Bound

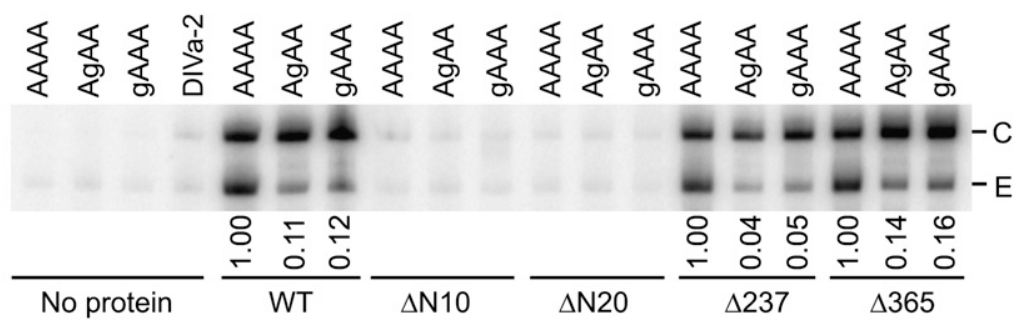

FIGURE 7. Equilibrium- and competition-binding assays for wild-type and mutant LtrA proteins with $\mathrm{N}$ - or C-terminal truncations. $(A)$ Equilibrium-binding assays. ${ }^{32} \mathrm{P}$-labeled DIVa-1 RNA (5 pM) was incubated with increasing concentrations of wild-type and the indicated mutant LtrA proteins in $450 \mathrm{NMT}$ medium for $60 \mathrm{~min}$ at $30^{\circ} \mathrm{C}$ (see Materials and Methods). The mixture was then filtered through layered nitrocellulose and Hybond-N nylon membranes, and radioactive RNA bound to the filters was quantified by using a PhosphorImager. The percentage of input RNA bound was plotted as a function of protein concentrations, and the plots were fit by a hyperbola to obtain $K_{\mathrm{d}}$ values. Apparent $K_{\mathrm{d}} s \pm$ the standard error of the fit for the curves shown are indicated. Similar results were obtained in three repeats of the experiment. A fit by the Hill equation gave $n=1$, indicating that binding of LtrA to DIVa-1 RNA is not cooperative (data not shown). (B) Competition-binding assays. A mixture containing $100 \mathrm{nM}$ each of ${ }^{32}$ P-labeled DIVa-2 RNA (135 nt; control [C]) and wildtype or mutant DIVa-1 RNA (119 nt; experimental [E]) was incubated with limiting amounts of wild-type or the indicated mutant LtrA proteins $(20 \mathrm{nM})$ in $450 \mathrm{NMT}$ for $60 \mathrm{~min}$ at $30^{\circ} \mathrm{C}$. After filtering through nitrocellulose, the bound RNAs were recovered by phenol-CIA extraction and analyzed by electrophoresis in a denaturing $4 \%$ polyacrylamide gel, which was dried and quantified with a PhosphorImager. Input shows individual RNAs or the mixture processed in the same way but without the filtration step. The control (C) RNA is wild type; the experimental (E) RNA is indicated above each lane; and the protein is indicated below the lanes. The binding ratios calculated as described in the Materials and Methods are indicated below the lanes for competition assays.

the fold of regions A and B. Region D could also contribute to splicing the wild-type Ll.LtrB- $\triangle \mathrm{ORF}$ intron by binding other regions of the intron RNA in a DIVa-dependent manner.
Three other LtrA regions (Fig. 6, regions $\mathrm{C}, \mathrm{E}, \mathrm{F}$ ) were identified by unigenic evolution analyses as being required for splicing both the wild-type Ll.LtrB- $\Delta$ ORF and Ll.LtrB- $\Delta$ DIVa intron and are presumably required for LtrA binding to core regions of the intron RNA. Region C extends from RT-2 to RT-3, while regions $\mathrm{E}$ and F correspond to the two halves of the $\mathrm{X}$ domain separated by the group II intron RT-specific insertion ti. Because binding to the core is not assayed directly, we cannot distinguish whether all three regions function directly in RNA binding or whether some are required to stabilize other regions that bind the RNA directly. We note, however, that the second half of the $\mathrm{X}$ domain (region F) is a site of mutations that inhibit RNA splicing activity both in LtrA and in the aI2 IEP (Moran et al. 1994; Cui et al. 2004). Further, a mutation in this region of LtrA was found to inactivate splicing of the wild-type Ll.LtrB- $\Delta$ ORF intron without strongly decreasing binding affinity for the intron RNA, the phenotype expected for a mutation that disrupts a critical contact with the catalytic core without affecting DIVa binding (Cui et al. 2004).

Figure 8 shows the regions of LtrA identified as being required for DIVa and core region binding mapped onto a three-dimensional model of the protein. The latter was constructed previously by threading the LtrA sequence on the X-ray crystal structure of HIV-1 RT heterodimer, with one LtrA subunit (monomer $\alpha$ ) modeled on the catalytic p66 subunit and the other subunit (monomer $\beta$ ) modeled on the p51 subunit in order to include a dimer interface (Blocker et al. 2005). In the three-dimensional model, LtrA regions $A$ and B (red), which are required for DIVa binding, are seen to comprise contiguous segments of the extended fingers region of the LtrA protein, while region $\mathrm{D}$ (pink), which may enhance binding to DIVa, is also part of the fingers adjoining regions $\mathrm{A}$ and $\mathrm{B}$ (Fig. 8A,B). The location of region $\mathrm{D}$ is consistent with the possibility that it stabilizes the fold of regions A and/or B. Regions C, E, and $\mathrm{F}$ (dark blue), identified as being involved in binding 
A

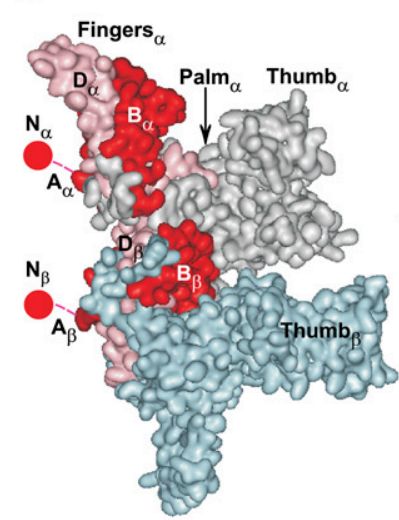

C

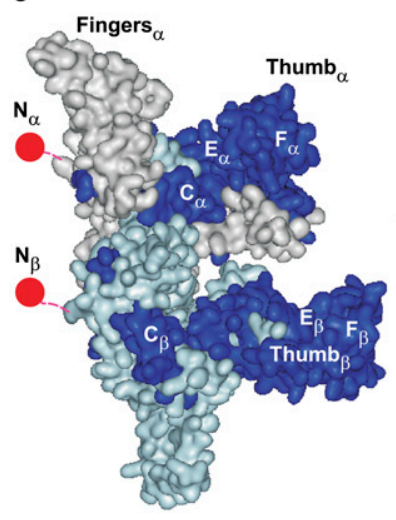

B

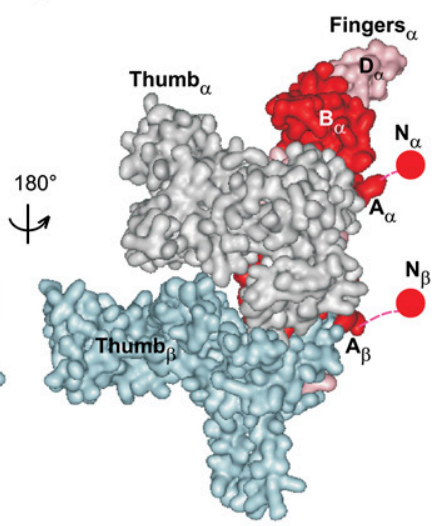

D

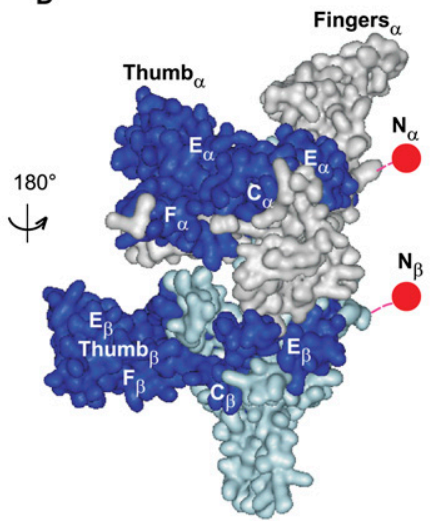

FIGURE 8. Three-dimensional model of LtrA highlighting regions required for binding of DIVa and intron RNA core regions. $(A, B)$ Front and back views of the model with regions $A$ and $B$ identified as being required for DIVa binding by unigenic evolution analysis (Fig. 3) shown in red. Region D, which may enhance LtrA binding to DIVa by stabilizing the fold of regions A and/or B (see text), is shown in pink. $(C, D)$ Front and back views of the model showing regions of LtrA required for Ll.LtrB- $\Delta$ DIVa intron splicing (Fig. 6) highlighted in dark blue. The LtrA subunit modeled on HIV-1 RT p66 (subunit $\alpha$ ) is in light gray, and the LtrA subunit modeled on HIV-1 p51 (subunit $\beta$ ) is in light cyan. The model encompasses LtrA amino acid residues 37-538. The N-terminal 36 amino acid residues, which could not be modeled based on HIV-1 RT, are represented as spheres at the $\mathrm{N}$-termini of the monomers ( $\mathrm{N} \alpha$ and $\mathrm{N} \beta$, respectively). Abbreviations: (A-F) regions defined by unigenic evolution analyses in Figures 3 and 6 , with subscripts $\alpha$ and $\beta$ referring to the subunit.

core regions of the intron RNA, form another contiguous surface that encompasses other parts of the fingers and most of the thumb (Fig. 8C,D). The model suggests that a large surface of the LtrA protein contributes directly or indirectly to intron RNA binding, with regions required for binding DIVa and intron RNA core regions falling neatly in separate parts of the molecule.

Two of our findings provide further insight into the structural organization of LtrA. First, the finding that N-terminal fragments of LtrA that end just after RT-4 or RT-7 bind specifically to DIVa (Figs. 4,7 ) indicates that all or part of these N-terminal segments can fold independently of other regions of the protein. These N-terminal fragments

of LtrA and their complexes with DIVa may be useful for structural analysis. Second, the finding that an N-terminal truncation of only 10 amino acid residues abolishes DIVa binding, while leaving relatively high RT and RNA splicing activity, suggests that all or part of the N-terminal extension upstream of RT-0 is a functionally distinct region required primarily for DIVa binding. This functionally distinct region may be relatively small, as larger $\mathrm{N}$-terminal truncations that extend farther (30 or 40 amino acids into RT-0) abolish both RT and RNA splicing activity (Cui et al. 2004).

LtrA's N-terminal extension upstream of RT-0, which is implicated here in DIVa binding, contains two predicted $\alpha$-helices that are conserved in position and show some sequence conservation in the N-terminal extensions of many, but not all group II intron IEPs (Blocker et al. 2005). Notable exceptions, which do not have such an N-terminal extension, include the Sinorhizobium melilotti group II intron RmInt1 and other bacterial lineage IID IEPs, raising the possibility that they interact differently with the intron RNA (Blocker et al. 2005). Non-LTRretrotransposon RTs also appear to have an N-terminal extension of the RT domain upstream of RT-0, but this region does not seem to be structurally related to the N-terminal extension of group II intron RTs, and its boundaries are unclear due to the presence of additional N-terminal domains that are not present in group II intron RTs (Malik et al. 1999; Blocker et al. 2005).

Notably, we find that base substitutions in the terminal tetraloop of DIVa that abolish LtrA binding in vitro have less effect in vivo, so that larger changes in the RNA were required to disrupt the interaction (Fig. 2). This difference presumably reflects that the high salt concentrations used to maintain specificity in the in vitro assays minimize the contribution of ionic interactions with the phosphate backbone, making binding more dependent upon the recognition of specific bases. These ionic interactions appear to make a greater contribution to the strength of binding at physiological salt concentrations in vivo, so that small mutations in the terminal tetraloop have less effect.

Considered together with previous findings, our results suggest a model in which the N-terminal extension of nascent LtrA binds DIVa soon after translation and nucleates further interactions between other regions of LtrA and core regions of the intron RNA. The binding of the $\mathrm{N}$ terminus of the nascent protein to a proximate RNA structure that encodes it readily accounts for LtrA's cis preference for splicing in vivo (Cui et al. 2004). Immediate binding to the intron RNA also stabilizes the LtrA protein, which is otherwise prone to irreversible denaturation (Saldanha et al. 1999). As noted previously, LtrA is largely monomeric in solution but binds the intron RNA with a stoichiometry of $2: 1$, suggesting that it functions in splicing as a dimer (Saldanha et al. 1999; Rambo and Doudna 2004). Our results suggest that a newly synthesized molecule of LtrA 
may be unable to bind DIVa until at least after translation of RT-4, which may be required for folding of N-terminal regions that directly contact DIVa. This protein-folding requirement may provide a built-in delay that enables a second molecule of LtrA to be translated from the same transcript before occlusion of the SD sequence. This second LtrA molecule may then bind the newly formed RNP containing the first LtrA molecule, accounting for the observed binding stoichiometry of 2:1. An alternate possibility is that the protein must dimerize prior to DIVa binding. The tight binding of one LtrA monomer to DIVa may provide an anchor enabling the protein to remain flexibly attached to the intron RNA as it undergoes conformational changes during different steps of splicing and then switches to initiation of reverse transcription during intron mobility. Indeed, the first translated LtrA molecule, which binds DIVa and makes other immediate RNA contacts, may be more strongly engaged with the RNA than the second, leaving the latter free to bind DNA target sites and carry out reverse transcription during intron mobility. Features of this model may also be relevant to non-LTRretrotransposon RTs, which, like group II intron RTs, appear to use N-terminal regions of the RT domain for specific binding of RNA templates (Bibillo and Eickbush 2002b), show a cis preference for acting on the RNA molecule that encodes them (Kulpa and Moran 2006), and appear to be monomers in solution but bind RNA templates as dimers (Yang and Eickbush 1998).

\section{MATERIALS AND METHODS}

\section{E. coli strains and growth conditions}

E. coli strain DH5 $\alpha$ was used for GFP fluorescence assays and unigenic evolution analysis with $\mathrm{pBDG}$ plasmids $\left(\mathrm{P}_{\mathrm{BAD}}\right.$ promoter), and HMS174(DE3) (Novagen) was used for GFP fluorescence assays and unigenic evolution analysis with pELG2 plasmids (T7lac promoter). BL21(DE3) and BL21(DE3)-R3/Rosetta (Novagen) were used for LtrA protein expression (Saldanha et al. 1999), and DH5 $\alpha$, $\mathrm{DH} 10 \mathrm{~B}$, and OmniMAX 2-T1 ${ }^{\mathrm{R}}$ cells (Invitrogen) were used for plasmid DNA preparation and cloning. Cells were grown in LB medium $(0.5 \%$ yeast extract, $1 \%$ peptone, $1 \% \mathrm{NaCl}$ at $\mathrm{pH} 7.5)$, with $2 \%$ Difco agar added for solid medium. Ampicillin and chloramphenicol were added at final concentrations of $100 \mu \mathrm{g} / \mathrm{mL}$ and $25 \mu \mathrm{g} / \mathrm{mL}$, respectively.

\section{Recombinant plasmids}

Plasmid pBDG-LtrA uses an arabinose-inducible promoter to express the LtrA protein, followed by a cassette consisting of DIVa with the SD sequence, the ATG initiation codon, and the next 14 codons of the LtrA ORF linked in-frame to codon 2 of GFP, and pBDG is the identical plasmid without the LtrA ORF. These plasmids are derivatives of pACYC184 (Fermentas), which carries a $\mathrm{cam}^{R}$ marker and contains a polylinker with XbaI, ClaI, HindIII, and EcoRV sites used in the constructions below. To construct the plasmids, the GFP coding sequence was PCR amplified from
pGFPuv (Clontech) by using the forward primer GFP/F (5' AAAAGCTTAAACTGCAGAGTAAAGGAGAAGAACTTTTCACT GGAG-3') and the reverse primer GFP/R (5'-GACGATATCTTA TTTGTAGAGCTCATCCATGCCATG-3'), and then digested with HindIII and EcoRV and cloned between the corresponding sites in the pACYC184 polylinker. This insertion disrupts the tet ${ }^{R}$ gene of pACYC184, and the GFP/F primer introduces HindIII and PstI sites for subsequent cloning steps. Second, a segment of the Ll.LtrB- $\Delta$ ORF intron containing DIVa (Ll.LtrB intron positions 518-605/2383-2395) was PCR amplified from pDIVa-1 (Singh et al. 2002) by using the primers pDIVa/F $\left(5^{\prime}\right.$-AAAAAGCTTG GGGTACAGTTATTGTGTAC- $\left.3^{\prime}\right)$ and pDIVa/R (5'-ACTCTGCA GGTTATTGTTCGTTATTC- $3^{\prime}$ ) and then cloned upstream of the GFP coding sequence between the newly introduced HindIII and PstI sites (Fig. 1B). Third, the regulator AraC and the $\mathrm{P}_{\mathrm{BAD}}$ arabinose-inducible promoter were PCR amplified from pACDIVa-lacZ (Singh et al. 2002) with primers pBAD/F (5'-ACTTCT AGATTATGACAACTTGACGGCTACATCATTC- $3^{\prime}$ ) and pBAD/R (5'-AAAATCGATGTTAGCCCAAAAAAACGGGTATGGAG-3') and cloned between the $\mathrm{XbaI}$ and $\mathrm{ClaI}$ sites to yield plasmid pBDG. Finally, the LtrA coding sequence was PCR amplified from pImp-1P (Saldanha et al. 1999) with primers LtrA/F (5'-AACATCGATAGG GAGGAAAACCTCAAAATGAAACC- $3^{\prime}$ ), which introduces a SD sequence and initiation codon, and LtrA/R ( $5^{\prime}$-ACTAAGCTTTCA CTTGTGTTTATGAATCACGTGACG-3'), and cloned between the ClaI and HindIII sites of pBDG (Fig. 1A) to yield pBDG-LtrA. Mutant derivatives of pBDG-LtrA with mutations in DIVa were made via PCR with primers that introduce the mutations.

A derivative of pBDG-LtrA with the N-terminal truncation $\Delta$ N10 was constructed by PCR of pBDG-LtrA with primers 5'-N10-ClaI-2 (5'-ACATCGATAGGGAGGAAAACCTCAAAATGAT CAGTAAAAATTCACAAGAAAATATAGACGAAGTTTTTACAA GAC-3') and 3' -pBDG-HindIII-2 (5'-AATAAAGCTTTCACTTG TGTTTATGAATCACGTGACGATGACAATGAAAGCATAC-3'), and then swapping the PCR product for the $1.8-\mathrm{kb}$ ClaI + HindIII fragment of that plasmid. Derivatives of pBDG-LtrA with different C-terminal truncations of LtrA were constructed by PCR using the forward primer LtrA-MutF (5'-GCTAACATCGATAGGGAGGA AAACCTCAAAATG-3') with reverse primers that introduce tandem TGA and TAA stop codons at the desired position, followed by a HindIII site. The PCR products were then digested with ClaI and HindIII and cloned between the corresponding sites of pBDG-LtrA in place of the wild-type LtrA ORF.

Plasmid pImp-1P, used for expression of the wild-type LtrA protein, contains the LtrA ORF cloned downstream from a tac promoter and $\phi 10$ SD sequence in the protein-expression vector pCYB02 (New England Biolabs) (Saldanha et al. 1999). LtrA is expressed from this plasmid as a fusion protein with a C-terminal tag containing an intein-linked chitin-binding domain, enabling LtrA purification via a chitin-affinity column, followed by inteincleavage. Derivatives of pImp-1P that express the $\mathrm{N}$-terminally truncated proteins $\mathrm{LtrA} / \Delta \mathrm{N} 10$ and $\mathrm{LtrA} / \Delta \mathrm{N} 20$ were described previously (Cui et al. 2004). Derivatives of Imp-1P that express C-terminally truncated LtrA proteins were constructed essentially as described above for $\mathrm{pBDG}$-LtrA C-terminal truncations.

pDIVa-1 and pDIVa-2 contain different lengths of the DIV + DIVa region of the Ll.LtrB- $\Delta$ ORF intron cloned downstream from a T7 promoter in pUC19 (Singh et al. 2002). Derivatives of pDIVa-1 with tetraloop AgAA and gAAA mutations were made with a QuickChange site-directed mutagenesis kit (Stratagene). 
pELG2-WT uses a T7lac promoter to express the wild-type Ll.LtrB- $\Delta$ ORF intron and flanking exons with the $3^{\prime}$ exon linked in frame to GFP, and pELG2- $\triangle$ DIVa is a derivative that contains the Ll.LtrB- $\Delta$ DIVa intron (previously denoted Ll.LtrB- $\Delta$ ORF/ $\Delta$ DIVa) (Cui et al. 2004). All constructs were confirmed by sequencing regions that had been subject to PCR.

\section{FACS assay for GFP expression}

For FACS assays, colonies were inoculated into $1 \mathrm{~mL}$ of $\mathrm{LB}$ medium containing chloramphenicol and grown overnight at $37^{\circ} \mathrm{C}$. A portion $(10 \mu \mathrm{L})$ of the overnight culture was then inoculated into $1-\mathrm{mL}$ fresh medium, grown with shaking at $30^{\circ} \mathrm{C}$ to $\mathrm{A}_{595}=0.4$, and induced with $1 \% \mathrm{~L}$-arabinose (SigmaAldrich) overnight. Cells were collected by centrifugation, resuspended in PBS (140 mM NaCl, $2.7 \mathrm{mM} \mathrm{KCl}, 9 \mathrm{mM} \mathrm{Na}_{2} \mathrm{HPO}_{4}$, $1.6 \mathrm{mM} \mathrm{KH} \mathrm{KO}_{4}$ at $\mathrm{pH}$ 7.4), and analyzed by using a FACS Caliber (Becton Dickinson, Immunocytometry Systems), with filter FL1 $(530 \pm 30 \mathrm{~nm})$. The data were analyzed with the CELLQuest program (Becton Dickinson). FACS assays for pELG2 plasmids were done as described (Cui et al. 2004).

\section{Colony-based fluorescence and immunoblot assays for GFP expression}

For colony fluorescence assays, E. coli $\mathrm{DH} 5 \alpha$ transformed with pBDG-LtrA or its mutant derivatives were plated on LB agar containing chloramphenicol and incubated overnight at $37^{\circ} \mathrm{C}$. The resulting colonies were lifted onto a nitrocellulose membrane filter (S-Pak; Millipore), which was then incubated overnight at $30^{\circ} \mathrm{C}$ on a petripad (Millipore) saturated with LB medium containing chloramphenicol and $1 \% \mathrm{~L}$-arabinose to induce plasmid transcription. The filters were scanned with a PhosphorImager (Typhoon Trio variable Mode Imager; GE Healthcare), using the fluorescence program with an excitation wavelength of $488 \mathrm{~nm}$ and a built-in 515-nm long-pass emission filter. Colonies that produce GFP appear as black dots against a light background.

For colony immunoblot assays, E. coli HMS174(DE3) containing pELG2- $\Delta$ DIVa or a pELG2- $\Delta$ DIVa library mutagenized for unigenic evolution analysis was plated on LB containing ampicillin at a density of 1200-1500 colonies per 150-mm diameter plate and incubated overnight at $37^{\circ} \mathrm{C}$. For library screening, the colonies on five to seven plates were lifted onto 35 -mm-diameter nitrocellulose filters, which were placed on pretripads saturated with LB medium containing ampicillin and $0.2 \mathrm{mM}$ isopropyl $\beta$-D-1-thiogalactopyranoside (IPTG), and incubated for $4 \mathrm{~h}$ at $37^{\circ} \mathrm{C}$ to induce transcription. The colonies were then lysed by incubating with drops of protoplast buffer $(15 \mathrm{mM}$ Tris- $\mathrm{HCl}$ at $\mathrm{pH}$ 8.0, $0.45 \mathrm{M}$ sucrose, $8 \mathrm{mM}$ EDTA, $0.4 \mathrm{mg} / \mathrm{mL}$ lysozyme [Sigma-Aldrich]) for $15 \mathrm{~min}$ at $4^{\circ} \mathrm{C}$, followed by lysis buffer $(10 \mathrm{mM}$ Tris- $\mathrm{HCl}$ at $\mathrm{pH} 8.0,10 \mathrm{mM} \mathrm{NaCl}, 1 \mathrm{mM}$ sodium citrate, $1.5 \% \mathrm{SDS}$ ) for $10 \mathrm{~min}$ at room temperature. The filters were washed twice in DNase I buffer $(40 \mathrm{mM}$ Tris- $\mathrm{HCl}$ at $\mathrm{pH} 7.5$, $10 \mathrm{mM} \mathrm{MgCl}_{2}$ ) at room temperature and incubated with drops containing DNase I (50 units/mL; New England Biolabs) in buffer at $37^{\circ} \mathrm{C}$ for $15 \mathrm{~min}$, then blocked by incubating in TTBS $(25 \mathrm{mM}$ Tris- $\mathrm{HCl}$ at $\mathrm{pH} 7.5,150 \mathrm{mM} \mathrm{NaCl}, 2.5 \mathrm{mM} \mathrm{KCl}, 0.5 \%$ Tween 20 ) containing 5\% dry milk and $1 \%$ bovine serum albumin (SigmaAldrich) at room temperature for $1 \mathrm{~h}$. After wiping away cell debris with a Kimwipe, the filters were incubated with primary
anti-GFP antibody (1:3,000 dilution; Clontech) for $2 \mathrm{~h}$ at room temperature, washed four times with TTBS at room temperature for $5 \mathrm{~min}$ each time, and incubated with HRP-conjugated goat anti-rabbit secondary antibody (Jackson Immunoresearch Laboratories) for $1 \mathrm{~h}$ at room temperature. Both the primary and secondary antibodies had been preadsorbed at room temperature for $0.5 \mathrm{~h}$ with a cell lysate from IPTG-induced E. coli HMS174(DE3) containing $\mathrm{pET}$, the vector backbone of pELG2- $\triangle \mathrm{DIV}$ (Cui et al. 2004). After washing the filters four times with TTBS for $5 \mathrm{~min}$ each time, they were developed by using the supersignal West Dura Extended Duration substrate (Pierce) and exposed to X-ray films. Splicing-competent colonies were identified by aligning the developed X-ray film and corresponding filters.

\section{RT-PCR assay of in vivo RNA splicing}

RT-PCR to detect ligated-exon RNAs was carried out on total cellular RNA that was isolated as described (Belfort et al. 1990) and further purified with a RNeasy mini kit (Qiagen). For the initial reverse transcription, $5 \mu \mathrm{g}$ of RNA was mixed with $1 \mathrm{pmol}$ of primer PPI ( $5^{\prime}$-GTTCTTCTCCTTTACTC-3') in $50 \mathrm{mM}$ Tris$\mathrm{HCl}$ ( $\mathrm{pH} 7.5$ ), $60 \mathrm{mM} \mathrm{NaCl}, 10 \mathrm{mM}$ DTT, and annealed by heating to $60^{\circ} \mathrm{C}$ for $3 \mathrm{~min}$, then chilling on ice. After additions to give $1.8 \mathrm{mM} \mathrm{MgCl}_{2}$ and $125 \mu \mathrm{M}$ of each dNTP, the reaction was initiated by adding M-MLV RT ( $6 \mathrm{U}$; Invitrogen), incubated at $48^{\circ} \mathrm{C}$ for $30 \mathrm{~min}$, and terminated by adding EDTA to $10 \mathrm{mM}$. A total of $1 / 20$ of the product was then used as a template for PCR in $50 \mu \mathrm{L}$ of reaction medium containing $2.5 \mathrm{U}$ of Taq DNA polymerase (Invitrogen), $150 \mu \mathrm{M}$ dNTPs, $3.5 \mathrm{mM} \mathrm{MgCl}_{2}$, $1 \mathrm{X}$ PCR buffer, and 25 pmoles each of primers PPI (see above) and PHIII ( $5^{\prime}$-CATATGGCTAGCATGACTGG-3') for 16 cycles of $94^{\circ} \mathrm{C}$ for 30 $\mathrm{sec}, 50^{\circ} \mathrm{C}$ for $30 \mathrm{sec}$, and $72^{\circ} \mathrm{C}$ for $1.5 \mathrm{~min}$. The products were analyzed in a $1.0 \%$ agarose gel containing ethidium bromide $(0.5 \mu \mathrm{g} / \mathrm{mL})$.

\section{Unigenic evolution analysis}

A pBDG-LtrA library containing random mutations in LtrA was constructed as described (Deminoff et al. 1995; Cui et al. 2004; Mohr et al. 2008) by using a GeneMorph II random mutagenesis kit (Stratagene) for mutagenic PCR. The PCR was done in $50 \mu \mathrm{L}$ of reaction medium containing $100 \mathrm{ng}$ of $\mathrm{pBDG}$-LtrA template, $125 \mathrm{ng}$ of each of primers LtrA-mutF (see above), and LtrAMutR2 (5'-CAATTTTTAATTTTAGTACACAATAAAGCTTTC AC-3'), $0.39 \mathrm{mM}$ dATP, $0.8 \mathrm{mM}$ dNTP mix, and $2.5 \mathrm{U}$ of Mutazyme II DNA polymerase. The primer LtrA-MutF introduces a ClaI site and a SD sequence, and the primer LtrA-MutR2 introduces a HindIII site and a stop codon. The reaction conditions were initial denaturation at $95^{\circ} \mathrm{C}$ for $2 \mathrm{~min}$, followed by $35 \mathrm{cycles}$ of $95^{\circ} \mathrm{C}$ for $30 \mathrm{sec}, 55^{\circ} \mathrm{C}$ for $30 \mathrm{sec}$, and $72^{\circ} \mathrm{C}$ for $2 \mathrm{~min}$, with final extension at $72^{\circ} \mathrm{C}$ for $10 \mathrm{~min}$. The $1.8 \mathrm{-kb}$ PCR product containing the mutagenized LtrA ORF was then gelpurified, digested with $\mathrm{ClaI}$ and HindIII, and cloned between the corresponding sites of the gel-purified pBDG-LtrA vector backbone. The mutation frequency determined by sequencing 36 randomly selected clones from the library was $0.54 \%$, the ratio of transitions to transversions was 3.4, and the average number of amino acid substitutions per protein was 7.3.

For screening, the library was transformed into E. coli $\mathrm{DH} 5 \alpha$, and functional variants that could bind DIVa binding were identified by their $\mathrm{GFP}^{-}$phenotype in the colony-based fluorescence 
assay described above. To confirm the phenotype, the plasmids were isolated by a miniprep procedure from $\mathrm{GFP}^{-}$colonies and retransformed into E. coli DH5 $\alpha$ for FACS assays in parallel with pBDG, which lacks LtrA. Those plasmids that gave $<15 \%$ of the fluorescence increase of pBDG after induction with L-arabinose were sequenced to identify the mutations.

The mutability value $(M)$ in a 25 -amino acid residue sliding window was calculated by using the equation:

$$
M=\frac{f_{\mathrm{O}_{\mathrm{mis}}}}{f_{\mathrm{E}_{\mathrm{mis}}}}-1,
$$

where $f_{\mathrm{O}_{\text {mis }}}$ is the observed frequency of missense mutations, and $f_{\mathrm{E}_{\text {mis }}}$ is the expected frequency. The latter was calculated for each codon based on the probability of a single nucleotide change producing a missense mutation, correcting for the transition/ transversion ratio of 3.4 for the variants selected from the library. To refine the analysis, only nonconservative substitutions based on the similarity matrix of Henikoff and Henikoff (1992) were treated as missense and conservative substitutions based on that matrix were treated as silent. Negative mutability values indicate hypomutability, with a maximum value of -1 indicating no missense mutations in a given window. Positive values indicate hypermutability and were normalized by using the equation:

$$
\frac{1}{f_{\mathrm{E}_{\mathrm{mis}}}}-1,
$$

so that all mutability values fall between -1 and +1 .

$\chi^{2}$ square values were calculated by using the equation:

$$
\chi^{2}=\frac{\left[\mathrm{abs}\left(\mathrm{O}_{\mathrm{mis}}-\mathrm{E}_{\mathrm{mis}}\right)-0.5\right]^{2}}{\mathrm{E}_{\mathrm{mis}}}+\frac{\left[\mathrm{abs}\left(\mathrm{O}_{\mathrm{sil}}-\mathrm{E}_{\mathrm{sil}}\right)-0.5\right]^{2}}{\mathrm{E}_{\mathrm{sil}}},
$$

where "abs" indicates the absolute value, $\mathrm{O}_{\text {mis }}$ and $\mathrm{O}_{\text {sil }}$ are numbers of missense and silent mutations defined as above in a given region, and $\mathrm{E}_{\mathrm{mis}}$ and $\mathrm{E}_{\mathrm{sil}}$ are expected numbers of missense and silent mutations in that region.

For unigenic analysis of Ll.LtrB- $\Delta \mathrm{DIVa}$ intron splicing, a pELG2- $\triangle$ DIVa library containing random mutations in the LtrA ORF was constructed as described (Cui et al. 2004). The mutagenic PCR was done with 0.06 pmoles of pLI1 template (Matsuura et al. 1997) and 50 pmoles each of primers LtrA $5^{\prime}$-SpeI (5'-GGGACTAGTACTTTAAGAAGGAGATATACATATG- ${ }^{\prime}$ ) and LtrA $3^{\prime}$-ClaI (5'-GGGGGCATCGATGCCCTTCGTTCGTAAAAA TTCA-3') in $100 \mu \mathrm{L}$ of reaction medium containing $0.39 \mathrm{mM}$ dATP, $0.15 \mathrm{mM}$ dCTP, $1.17 \mathrm{mM}$ dGTP, $3.85 \mathrm{mM}$ dTTP, $11 \mathrm{mM}$ $\mathrm{MgCl}_{2}, 0.5 \mathrm{mM} \mathrm{MnCl}$, and $5 \mathrm{U}$ of Taq DNA polymerase (Invitrogen). The reaction conditions were initial denaturation at $94^{\circ} \mathrm{C}$ for $5 \mathrm{~min}$, followed by $16 \mathrm{cycles}$ at $91^{\circ} \mathrm{C}$ for $1 \mathrm{~min}, 51^{\circ} \mathrm{C}$ for $1 \mathrm{~min}$, and $72^{\circ} \mathrm{C}$ for $10 \mathrm{~min}$, with final extension at $72^{\circ} \mathrm{C}$ for $15 \mathrm{~min}$. The 1.8-kb PCR product containing the mutagenized LtrA ORF was then gel-purified, digested with SpeI and ClaI, and cloned between the corresponding sites of pELG1- $\triangle$ ORF (Cui et al. 2004). Sequencing of 48 randomly picked clones showed that the mutation rate was $0.6 \%$, and the ratio of transitions to transversions was 3.0.

For unigenic evolution analysis, the pELG2- $\Delta$ DIVa library was transformed into HMS174(DE3), and splicing-competent LtrA variants were identified by colony immunoblot assay (see above).
Individual colonies were then grown up in LB medium containing ampicillin, and plasmids encoding LtrA variants were isolated by a minprep procedure and transformed into HMS174(DE3) to retest their splicing phenotype by RT-PCR (see above). Variants that were positive for splicing in the RT-PCR assay were sequenced to identify changes in the LtrA ORF. The mutational data were analyzed as described above.

\section{Purification of wild-type and mutant LtrA proteins}

Wild-type and mutant LtrA proteins were expressed in E. coli BL21(DE3) or Rosetta (DE3) from the intein-based expression plasmid pImp-1P or its mutant derivatives. After autoinduction (Studier 2005), proteins were purified by using a chitin-affinity column, as described (Saldanha et al. 1999). The collected protein fractions were pooled and dialyzed against buffer A $(300 \mathrm{mM}$ $\mathrm{NaCl}, 25 \mathrm{mM}$ Tris- $\mathrm{HCl}$ at $\mathrm{pH}$ 7.5, $0.5 \mathrm{mM}$ EDTA, $2 \mathrm{mM}$ DTT, $10 \%$ glycerol) for $90 \mathrm{~min}$ and further purified via FPLC using a Hitrap heparin HP column (pack size $5 \mathrm{~mL}$; GE Healthcare) eluted with a gradient of $50 \mathrm{mM}$ to $2 \mathrm{M} \mathrm{NaCl}$ in buffer B $(25 \mathrm{mM}$ Tris- $\mathrm{HCl}$ at $\mathrm{pH}$ 7.5, $0.5 \mathrm{mM}$ EDTA, $2 \mathrm{mM}$ DTT, 10\% glycerol). Peak fractions were combined and concentrated by using an Amicon Ultra-4 filter (Millipore) with a cutoff of $10 \mathrm{kDa}$. After adding glycerol to $50 \%$, the proteins were stored at $4^{\circ} \mathrm{C}$ for shortterm use or at $-80^{\circ} \mathrm{C}$ for longer periods. Protein concentrations were determined either from $\mathrm{A}_{280}$ measurements by using an extinction coefficient calculated from the amino acid sequence (http:/www.basic.northwestern.edu/biotools/proteincalc.html) or by Bradford protein assay (Bio-Rad), using LtrA protein previously calibrated by $\mathrm{A}_{280}$ as a standard.

\section{SDS-PAGE and immunoblotting}

Purified proteins were analyzed by electrophoresis in a $0.1 \%$ SDS/ $10 \%$ polyacrylamide gel with a $4 \%$ polyacrylamide stacking gel. Protein bands were visualized by staining with Coomassie Blue. For immunoblot analysis, E. coli harboring pBDG-LtrA or its mutant derivatives were grown overnight at $37^{\circ} \mathrm{C}$, and $10 \mu \mathrm{L}$ of the overnight culture was then inoculated into $2-\mathrm{mL}$ LB medium containing $1 \% \mathrm{~L}$-arabinose and induced overnight at $30^{\circ} \mathrm{C}$. Equal amounts of cells (O.D.595 $=0.05$ ) were pelleted by microcentrifugation, and proteins were solubilized by adding an equal volume of $2 \mathrm{X}$ gel loading buffer $(100 \mathrm{mM}$ Tris- $\mathrm{HCl}$ at $\mathrm{pH} 6.8,200 \mathrm{mM}$ dithiothreitol, $4 \%$ [w/v] SDS, $0.2 \%$ bromophenol blue, $20 \%$ glycerol) and heating to $100^{\circ} \mathrm{C}$ for $10 \mathrm{~min}$ prior to SDS-PAGE as above. Immunoblotting was done with a rabbit anti-LtrA polyclonal antibody preparation essentially as described (Cui et al. 2004).

\section{Biochemical assays}

RNA-binding assays were done with small ${ }^{32} \mathrm{P}$-labeled RNAs containing wild-type or mutant DIVa (Singh et al. 2002). The RNAs were transcribed from HindIII-digested pDIVa-1 and pDIV-2 by using a T7 MEGAshortscript kit (Applied Biosystems/ Ambion). After digestion with Turbo DNase from the kit, the RNAs were purified by two passes through a Microspin G-25 column (GE Healthcare). Prior to use, the RNAs were renatured by heating to $92^{\circ} \mathrm{C}$ and slowly cooling to $30^{\circ} \mathrm{C}$ before adding reaction medium. The pDIVa-1 and pDIVa-2 transcripts are 119 and 135 nts, respectively. 
For equilibrium-binding assays, ${ }^{32} \mathrm{P}$-labeled DIVa-1 RNA (5 pM) was incubated with increasing concentrations of LtrA protein in $50 \mu \mathrm{L}$ of reaction medium containing $450 \mathrm{NMT}(450 \mathrm{mM} \mathrm{NaCl}$, $5 \mathrm{mM} \mathrm{MgCl}_{2}$, and $40 \mathrm{mM}$ Tris- $\mathrm{HCl}$ at $\mathrm{pH} 7.5$ ), and $100 \mu \mathrm{g} / \mathrm{mL}$ bovine serum albumin. After incubating for $1 \mathrm{~h}$ at $30^{\circ} \mathrm{C}$, the mixture was multichannel pipetted into a 96-well vacuum manifold and filtered through top nitrocellulose (Trans-Blot; Bio-Rad) and bottom nylon membranes filters (Hybond-N; GE Healthcare), which had been presoaked in 450 NMT. The RNA-protein complex binds to the nitrocellulose membrane, while the unbound RNA binds to the nylon membrane. The membranes were washed three times with $50 \mu \mathrm{L}$ of $450 \mathrm{NMT}$, dried, and scanned with a PhosphorImager (Typhoon Trio variable Mode Imager, GE Healthcare). Spot intensities were quantified using ImageQuant software TL-v2005 software (GE Healthcare). The percentage of input RNA bound was plotted as a function of protein concentrations, and the plots were fit by a hyperbola to obtain $K_{\mathrm{d}}$ 's.

For competition-binding assays, a mixture containing $100 \mathrm{nM}$ of each RNA was renatured as described above, then incubated with $20 \mathrm{nM}$ LtrA protein in $100 \mu \mathrm{L}$ of $450 \mathrm{NMT}$ for $60 \mathrm{~min}$ at $30^{\circ} \mathrm{C}$, and filtered through nitrocellulose (Protran BA 85, pore size $=0.45 \mu \mathrm{M}, \phi=25 \mathrm{~mm}$; Sigma-Aldrich) to retain proteinbound RNA. The RNAs were eluted from the filter by phenol-CIA extraction $(200 \mu \mathrm{L}$ of $10 \mathrm{mM}$ Tris- $\mathrm{HCl}$ at $\mathrm{pH} 7.5,1 \mathrm{mM}$ EDTA plus $600 \mu \mathrm{L}$ of phenol-CIA), followed by ethanol-precipitation in the presence of $1 / 10(\mathrm{v} / \mathrm{v}) 5 \mathrm{M}$ ammonium acetate $(\mathrm{pH} 5.3)$, and $100 \mu \mathrm{g}$ of glycogen carrier (Roche). After centrifugation, the pellet was dissolved in $10 \mu \mathrm{L}$ of water, mixed with an equal amount of RNA loading dye, and then analyzed in a denaturing polyacrylamide gel (4\% acylamide/bisacrylamide [19:1 (w/w)], 8.0 M urea in $89 \mathrm{mM}$ Tris, $89 \mathrm{mM}$ boric acid, $2 \mathrm{mM}$ EDTA at $\mathrm{pH} 8.3$ ). The gel was dried and quantified using a PhosphorImager as above. The binding ratio was defined as the ratio of the mutant to wildtype RNA bound by the protein relative to the input ratio of the RNAs. RT and RNA-splicing assays were done as described in the Legends of Supplemental Figures S6 and S7.

\section{SUPPLEMENTAL MATERIAL}

Supplemental material can be found at http://www.rnajournal.org.

\section{ACKNOWLEDGMENTS}

We thank Georg Mohr for comments on the manuscript. This work was supported by NIH grant R01 GM037951.

Received November 16, 2009; accepted December 22, 2009.

\section{REFERENCES}

Belfort M, Ehrenman K, Chandry PS. 1990. Genetic and molecular analysis of RNA splicing in Escherichia coli. Methods Enzymol 181: $521-539$.

Bibillo A, Eickbush TH. 2002a. High processivity of the reverse transcriptase from a non-long-terminal repeat retrotransposon. J Biol Chem 277: 34836-34845.

Bibillo A, Eickbush TH. 2002b. The reverse transcriptase of the R2 non-LTR retrotransposon: Continuous synthesis of cDNA on non-continuous RNA templates. J Mol Biol 316: 459-473.
Blocker FJ, Mohr G, Conlan LH, Qi L, Belfort M, Lambowitz AM. 2005. Domain structure and three-dimensional model of a group II intron-encoded reverse transcriptase. RNA 11: 14-28.

Carignani G, Groudinsky O, Frezza D, Schiavon E, Bergantino E, Slonimski PP. 1983. An mRNA maturase is encoded by the first intron of the mitochondrial gene for the subunit I of cytochrome oxidase in S. cerevisiae. Cell 35: 733-742.

Cech TR. 1986. The generality of self-splicing RNA: Relationship to nuclear mRNA splicing. Cell 44: 207-210.

Chen B, Lambowitz AM. 1997. De novo and DNA primer-mediated initiation of cDNA synthesis by the Mauriceville retroplasmid reverse transcriptase involve recognition of a $3^{\prime}$ CCA sequence. J Mol Biol 271: 311-332.

Cui X, Matsuura M, Wang Q, Ma H, Lambowitz AM. 2004. A group II intron-encoded maturase functions preferentially in cis and requires both the reverse transcriptase and $\mathrm{X}$ domains to promote RNA splicing. J Mol Biol 340: 211-231.

Dai L, Chai D, Gu SQ, Gabel J, Noskov SY, Blocker FJ, Lambowitz AM, Zimmerly S. 2008. A three-dimensional model of a group II intron RNA and its interaction with the intronencoded reverse transcriptase. Mol Cell 30: 472-485.

Deminoff SJ, Tornow J, Santangelo GM. 1995. Unigenic evolution: A novel genetic method localizes a putative leucine zipper that mediates dimerization of the Saccharomyces cerevisiae regulator Gcrlp. Genetics 141: 1263-1274.

D'Souza LM, Zhong J. 2002. Mutations in the Lactococcus lactis Ll.LtrB group II intron that retain mobility in vivo. BMC Mol Biol 3: 17. doi: 10.1186/1471-2199-3-17.

Guzman LM, Belin D, Carson MJ, Beckwith J. 1995. Tight regulation, modulation, and high-level expression by vectors containing the arabinose PBAD promoter. J Bacteriol 177: 4121-4130.

Henikoff S, Henikoff JG. 1992. Amino acid substitution matrices from protein blocks. Proc Natl Acad Sci USA 89: 10915-10919.

Huang HR, Chao MY, Armstrong B, Wang Y, Lambowitz AM, Perlman PS. 2003. The DIVa maturase binding site in the yeast group II intron aI2 is essential for intron homing but not for in vivo splicing. Mol Cell Biol 23: 8809-8819.

Kulpa KA, Moran JV. 2006. Cis-preferential LINE-1 reverse transcriptase activity in ribonucleoprotein particles. Nat Struct Mol Biol 13: 655-660.

Lambowitz AM, Zimmerly S. 2004. Mobile group II introns. Annu Rev Genet 38: 1-35.

Luan DD, Korman MH, Jakubczak JL, Eickbush TH. 1993. Reverse transcription of R2Bm RNA is primed by a nick at the chromosomal target site: A mechanism for non-LTR retrotransposition. Cell 72: 595-605.

Madhani HD, Guthrie C. 1992. A novel base-pairing interaction between U2 and U6 snRNAs suggests a mechanism for the catalytic activation of the spliceosome. Cell 71: 803-817.

Malik HS, Burke WD, Eickbush TH. 1999. The age and evolution of non-LTR retrotransposable elements. Mol Biol Evol 16: 793-805.

Matsuura M, Saldanha R, Ma H, Wank H, Yang J, Mohr G, Cavanagh S, Dunny GM, Belfort M, Lambowitz AM. 1997. A bacterial group II intron encoding reverse transcriptase, maturase, and DNA endonuclease activities: Biochemical demonstration of maturase activity and insertion of new genetic information within the intron. Genes \& Dev 11: 2910-2924.

Matsuura M, Noah JW, Lambowitz AM. 2001. Mechanism of maturase-promoted group II intron splicing. EMBO J 20: 72597270 .

Mohr G, Del Campo M, Mohr S, Yang Q, Jia H, Jankowsky E, Lambowitz AM. 2008. Function of the C-terminal domain of the DEAD-box protein Mss116p analyzed in vivo and in vitro. J Mol Biol 375: 1344-1364.

Moran JV, Mecklenburg KL, Sass P, Belcher SM, Mahnke D, Lewin A, Perlman P. 1994. Splicing defective mutants of the COXI gene of yeast mitochondrial DNA: Initial definition of the maturase domain of the group II intron AI2. Nucleic Acids Res 22: 20572064. 
Peebles CL, Perlman PS, Mecklenburg KL, Petrillo ML, Tabor JH, Jarrell KA, Cheng HL. 1986. A self-splicing RNA excises an intron lariat. Cell 44: 213-223.

Pyle AM, Lambowitz AM. 2006. Group II introns: Ribozymes that splice RNA and invade DNA. In The RNA world (ed. RF Gesteland et al.), 3rd ed., pp 469-505. Cold Spring Harbor Laboratory Press, Cold Spring Harbor, NY.

Rambo RP, Doudna JA. 2004. Assembly of an active group II intronmaturase complex by protein dimerization. Biochemistry 43: 6486-6497.

Saldanha R, Chen B, Wank H, Matsuura M, Edwards J, Lambowitz AM. 1999. RNA and protein catalysis in group II intron splicing and mobility reactions using purified components. Biochemistry 38: 9069-9083.

San Filippo J, Lambowitz AM. 2002. Charaterization of the C-terminal DNA-binding/DNA endonuclease region of a group II intronencoded protein. J Mol Biol 324: 933-951.

Sharp PA. 1985. On the origin of RNA splicing and introns. Cell 42: 397-400.

Sharp PA. 1991. Five easy pieces. Science 254: 663. doi: 10.1126/ science. 1948046.

Shukla GC, Padgett RA. 2002. A catalytically active group II intron domain 5 can function in the U12-dependent spliceosome. Mol Cell 9: 1145-1150.

Singh RN, Saldanha RJ, D'Souza LM, Lambowitz AM. 2002. Binding of a group II intron-encoded reverse transcriptase/maturase to its high affinity intron RNA binding site involves sequence-specific recognition and autoregulates translation. J Mol Biol 318: 287-303.

Studier FW. 2005. Protein production by auto-induction in high density shaking cultures. Protein Expr Purif 41: 207-234.

Toor N, Keating KS, Taylor SD, Pyle AM. 2008. Crystal structure of a self-spliced group II intron. Science 320: 77-82.

Toor N, Keating KS, Fedorova O, Rajashankar K, Wang J, Pyle AM. 2010. Tertiary architecture of the Oceanobacillus iheyensis group II intron. RNA 16: 57-69.

Wank H, San Filippo J, Singh RN, Matsuura M, Lambowitz AM. 1999. A reverse transcriptase/maturase promotes splicing by binding at its own coding segment in a group II intron RNA. Mol Cell 4: 239-250.

Watanabe K, Lambowitz AM. 2004. High-affinity binding site for a group II intron-encoded reverse transcriptase/maturase within a stem-loop structure in the intron RNA. RNA 10: 1433-1443.

Xiong Y, Eickbush TH. 1990. Origin and evolution of retroelements based upon their reverse transcriptase sequences. EMBO J 9: 33533362.

Yang J, Eickbush TH. 1998. RNA-induced changes in the activity of the endonuclease encoded by the R2 retrotransposable element. Mol Cell Biol 18: 3455-3465.

Zimmerly S, Guo H, Perlman PS, Lambowitz AM. 1995. Group II intron mobility occurs by target DNA-primed reverse transcription. Cell 83: 545-554. 

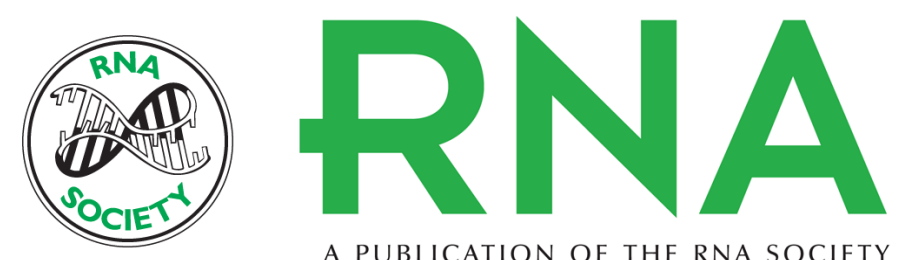

A PUBLICATION OF THE RNA SOCIETY

\section{Genetic identification of potential RNA-binding regions in a group II intron-encoded reverse transcriptase}

Shan-Qing Gu, Xiaoxia Cui, Sijiong Mou, et al.

RNA 2010 16: $732-747$ originally published online February 23, 2010

Access the most recent version at doi:10.1261/rna.2007310

\section{Supplemental http://rnajournal.cshlp.org/content/suppl/2010/02/01/rna.2007310.DC1 \\ Material}

References This article cites 39 articles, 12 of which can be accessed free at: http://rnajournal.cshlp.org/content/16/4/732.full.html\#ref-list-1

\section{License}

Email Alerting Receive free email alerts when new articles cite this article - sign up in the box at the Service top right corner of the article or click here.

\section{IIII!" Providing Precise Solutions for your research.}

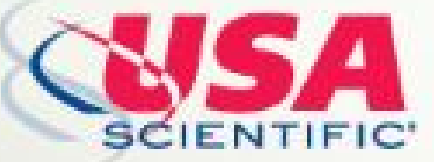

To subscribe to $R N A$ go to:

http://rnajournal.cshlp.org/subscriptions 